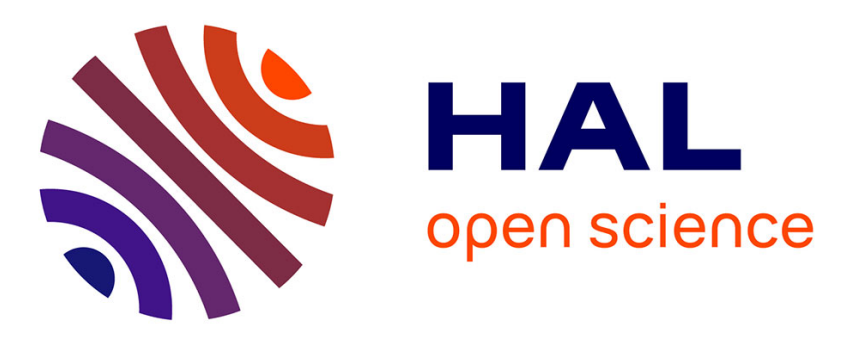

\title{
A very accurate Arbitrary Lagrangian-Eulerian meshless method for Computational Aeroacoustics
}

Luis Ramírez, Xesús Nogueira, Sofiane Khelladi, Abdelkader Krimi, Ignasi Colominas

\section{- To cite this version:}

Luis Ramírez, Xesús Nogueira, Sofiane Khelladi, Abdelkader Krimi, Ignasi Colominas. A very accurate Arbitrary Lagrangian-Eulerian meshless method for Computational Aeroacoustics. Computer Methods in Applied Mechanics and Engineering, 2018, 342, pp.116-141. 10.1016/j.cma.2018.07.036 . hal-02438523

\section{HAL Id: hal-02438523 \\ https://hal.science/hal-02438523}

Submitted on 14 Jan 2020

HAL is a multi-disciplinary open access archive for the deposit and dissemination of scientific research documents, whether they are published or not. The documents may come from teaching and research institutions in France or abroad, or from public or private research centers.
L'archive ouverte pluridisciplinaire HAL, est destinée au dépôt et à la diffusion de documents scientifiques de niveau recherche, publiés ou non, émanant des établissements d'enseignement et de recherche français ou étrangers, des laboratoires publics ou privés. 


\title{
A very accurate Arbitrary Lagrangian-Eulerian meshless method for Computational Aeroacoustics
}

\author{
Luis Ramírez ${ }^{a}, *$ Xesús Nogueira ${ }^{a}$, Sofiane Khelladi ${ }^{b}$, Abdelkader Krimi $^{c}$, \\ Ignasi Colominas $^{\mathrm{a}}$ \\ ${ }^{a}$ Universidade da Coruña,Group of Numerical Methods in Engineering, Campus de Elviña, 15071, A Coruña, Spain \\ ${ }^{\mathrm{b}}$ Laboratoire de Dynamique des Fluides, Arts et Métiers ParisTech, 151 Boulevard de l'Hôpital, 75013 Paris, France \\ ${ }^{\mathrm{c}}$ Institut de Recherche en Constructibilité, École Spéciale des Travaux Publics, 28 Avenue du Président Wilson, 94230 Cachan, France
}

\begin{abstract}
In this work, we propose a new meshless approach based on a Galerkin discretization of a set of conservation equations on an Arbitrary Lagrangian-Eulerian framework. In particular, we solve the Linearized Euler Equations, using Moving Least Squares as weight functions in the Galerkin discretization. Riemann solvers are introduced in the formulation for the discretization of the convective fluxes. Differently from a purely Lagrangian approach, as it is usual in SPH, the present method is able to work in both Eulerian and Lagrangian configurations, which allows using all the advantages of the Lagrangian approaches in the context of Computational Aeroacoustics.
\end{abstract}

Keywords: Meshless methods; Smoothed Particle Hydrodynamics; Moving Least Squares; Computational Aeroacoustics

\section{Introduction}

The low magnitude of acoustic waves and the wide range of frequencies involved in Aeroacoustics, make mandatory the use of very accurate low-dissipative numerical schemes for the simulation of these problems. The development of Computational Aeroacoustics (CAA) is related to the development of these kinds of numerical methods. Nowadays, the standard numerical techniques in CAA are mainly grid-based methods [1-7] although some approaches using meshless methods have also been presented [8-11].

The use of Lagrangian methods in the context of CAA may find interest for problems involving deformable or moving boundaries in the propagation media or multiphase flows, such as combustion noise, sound propagation through multiphase flows or bubble acoustics. However, there are very few Lagrangian numerical methods to solve

\footnotetext{
* Corresponding author.

E-mail address: luis.ramirez@udc.es (L. Ramírez).
} 
Computational Aeroacoustics problems in the literature. In particular, related to SPH methods the authors are only aware of the work of [12-15].

In this work, we propose a new meshless approach based on a Galerkin discretization of a set of conservation equations on an Arbitrary Lagrangian-Eulerian approach. In particular, we solve the Linearized Euler Equations, using Moving Least Squares (MLS) [16,17] as weight functions for the Galerkin discretization.

The proposed numerical method can be seen as a general formulation which includes some well-known meshfree methods as a particular case. Thus, on one hand it is shown that the presented discretization includes the SPH-ALE formulation [18-20], which is recovered with a particular choice of the weight functions. On the other hand, the proposed formulation can also be seen as a generalization of the Finite Point Method [21-23], which is recovered for Eulerian configurations with and homogeneous distribution of particles. The proposed method has also some similarities with that proposed in [24,25] for astrophysical applications. However, the numerical fluxes are treated here in a different manner.

One of the advantages of the present approach is that it is able to work in both Eulerian and Lagrangian configurations. Moreover, the use of MLS shape functions instead of the more usual particle approximations using kernel functions [18,26,27] leads to a decrease in the number of particles involved in the approximation, and also, in the context of SPH-Riemann methods, to a fewer number of Riemann problems to be solved. Moreover, the use of MLS functions verifies, by definition, the discrete partition of unity, and the exact zero-gradient condition for constant functions to be verified. Nevertheless, in addition to MLS, our approach stay open to the use of other reconstruction methods such as Radial Point Interpolation Method (RPIM) [28], Maximum Entropy Method (MaxEnt) [29] or Natural Element Method (NEM) [30,31].

The paper is organized as follows. First, we introduce the Linearized Euler equations in ALE form. Then, we introduce MLS approximations, and in Section 4 we present the proposed meshless discretization. The accuracy of the method is tested and validated in Section 5, and finally, the conclusions are drawn.

\section{Governing equations}

The three dimensional linearized Euler equations written in conservative form are:

$$
L_{w} \boldsymbol{U}+\nabla \cdot(\mathcal{F})+\mathcal{H}(\boldsymbol{U})=\boldsymbol{S}(\boldsymbol{U})
$$

where we define by $L_{\boldsymbol{w}}$ a Lagrangian operator which is expressed for a vector $\boldsymbol{U}$ as $L_{\boldsymbol{w}} \boldsymbol{U}=\partial_{t} \boldsymbol{U}+\nabla \cdot(\boldsymbol{w} \otimes \boldsymbol{U})$, and the vector $\boldsymbol{w}=\left(w_{1}, w_{2}, w_{3}\right)^{T}$ stands for the velocity of the Lagrangian frame. The source term is denoted by $\boldsymbol{S}(\boldsymbol{U})$. Moreover, $\boldsymbol{U}$ and $\mathcal{F}=\left(\mathcal{F}_{x}, \mathcal{F}_{y}, \mathcal{F}_{z}\right)$ are the vector of the conservative variables and the inviscid flux vector, namely

$$
\begin{aligned}
& \boldsymbol{U}=\left(\begin{array}{c}
\rho^{\prime} \\
\rho_{0} u^{\prime} \\
\rho_{0} v^{\prime} \\
\rho_{0} w^{\prime} \\
p^{\prime}
\end{array}\right) \\
& \mathcal{F}_{x}=\left.\left(\begin{array}{c}
\rho^{\prime}\left(u_{0}-w_{1}\right)+\rho_{0} u^{\prime} \\
\rho_{0} u^{\prime}\left(u_{0}-w_{1}\right)+p^{\prime} \\
\rho_{0} v^{\prime}\left(u_{0}-w_{1}\right) \\
\rho_{0} w^{\prime}\left(u_{0}-w_{1}\right) \\
p^{\prime}\left(u_{0}-w_{1}\right)+\gamma p_{0} u^{\prime}
\end{array}\right), \quad \begin{array}{c}
\rho^{\prime}\left(v_{0}-w_{2}\right)+\rho_{0} v^{\prime} \\
\rho_{0} u^{\prime}\left(v_{0}-w_{2}\right) \\
\rho_{0} v^{\prime}\left(v_{0}-w_{2}\right)+p^{\prime} \\
\rho_{0} w^{\prime}\left(v_{0}-w_{2}\right) \\
p^{\prime}\left(v_{0}-w_{2}\right)+\gamma p_{0} v^{\prime}
\end{array}\right), \\
& \mathcal{F}_{z}=\left(\begin{array}{c}
\rho^{\prime}\left(w_{0}-w_{3}\right)+\rho_{0} w^{\prime} \\
\rho_{0} u^{\prime}\left(w_{0}-w_{3}\right) \\
\rho_{0} v^{\prime}\left(w_{0}-w_{3}\right) \\
\rho_{0} w^{\prime}\left(w_{0}-w_{3}\right)+p^{\prime} \\
p^{\prime}\left(w_{0}-w_{3}\right)+\gamma p_{0} w^{\prime}
\end{array}\right)
\end{aligned}
$$

where the mean stationary solution is $\boldsymbol{U}_{0}=\left(\rho_{0}, \rho_{0} u_{0}, \rho_{0} v_{0}, \rho_{0} w_{0}, p_{0}\right)$. In the acoustic perturbations $\boldsymbol{U}, \rho^{\prime}$ is the fluctuating density, $\boldsymbol{u}^{\prime}=\left(u^{\prime}, v^{\prime}, w^{\prime}\right)^{T}$ the velocity and $p^{\prime}$ the pressure. Moreover, the term $\mathcal{H}(\boldsymbol{U})$ contains terms 


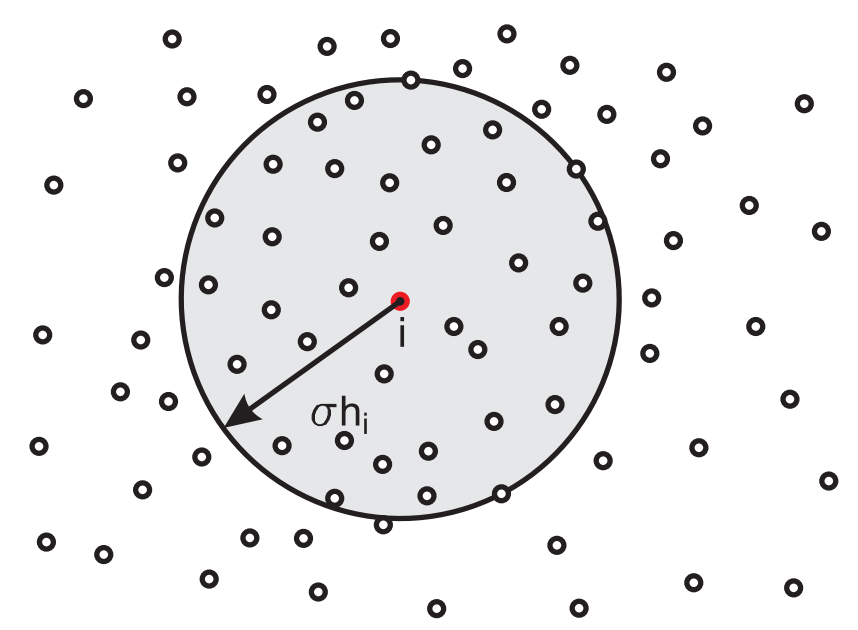

Fig. 1. Schematic representation of a particle $i$ and its neighbor particles inside the compact support defined by the smoothing length $h_{i}$ and the constant $\sigma$.

related to the gradients of the mean flow, which are zero when the mean flow is uniform

$$
\mathcal{H}(\boldsymbol{U})=\left(\begin{array}{c}
0 \\
\left(\rho_{0} \boldsymbol{u}^{\prime}+\rho^{\prime}\left(\boldsymbol{u}_{0}-\boldsymbol{w}\right)\right) \cdot \nabla u_{0} \\
\left(\rho_{0} \boldsymbol{u}^{\prime}+\rho^{\prime}\left(\boldsymbol{u}_{0}-\boldsymbol{w}\right)\right) \cdot \nabla v_{0} \\
\left(\rho_{0} \boldsymbol{u}^{\prime}+\rho^{\prime}\left(\boldsymbol{u}_{0}-\boldsymbol{w}\right)\right) \cdot \nabla w_{0} \\
(\gamma-1) p^{\prime} \nabla \cdot \boldsymbol{u}_{0}-(\gamma-1) u^{\prime} \nabla p_{0}
\end{array}\right) .
$$

\section{Interpolation operator: moving least squares reproducing kernel approximations}

In this work we derive a new meshless formulation, and we apply it to the Linearized Euler Equations. The new formulation is similar to that presented in [24,25] for astrophysics, but here we address the flux functions in a different manner. The numerical method is based on the use of MLS shape functions, and we refer the interested reader to [17] for a complete description of MLS approximations. Here, we briefly introduce the computation of MLS shape functions.

Let us consider a set of randomly distributed points. Each point $i$ has a position $\boldsymbol{r}_{i}$, and an associate value $\phi_{i}$. For now on, we call particle to a point with an associate value. Moreover, each particle $i$ has $n_{i}$ neighboring particles. These neighbor particles are defined as those particles that are inside the compact support of the particle, as schematically represented in Fig. 1. The compact support of a particle $i$ is defined by the smoothing length $h_{i}$.

Thus, if we consider a function $\phi(x)$ defined on a domain $\Omega$ (which in this framework is the compact support of the particle), the basic idea of the MLS approach is to approximate $\phi(x)$, at a given point $\boldsymbol{r}$, through a weighted least-squares fitting of $\phi(x)$ in a neighborhood of $\boldsymbol{r}$ as

$$
\phi(\boldsymbol{r}) \approx \widehat{\phi}(\boldsymbol{r})=\sum_{i=1}^{m} p_{i}(\boldsymbol{r}) \boldsymbol{\alpha}_{i}(\boldsymbol{r})=\boldsymbol{p}^{T}(\boldsymbol{r}) \boldsymbol{\alpha}(\boldsymbol{r})
$$

where $\boldsymbol{p}^{T}(\boldsymbol{r})=\left(1, x, y, x^{2}, y^{2}, x y, \ldots\right) \in \mathbb{R}^{m}$ is an $m$-dimensional basis, and $\boldsymbol{\alpha}(\boldsymbol{r})$ is a set of parameters to be determined, such that they minimize the following functional of error

$$
J(\boldsymbol{\alpha}(\boldsymbol{r}))=\int_{\boldsymbol{y} \in \Omega_{\boldsymbol{r}}} W\left(\boldsymbol{r}-\boldsymbol{r}^{\prime}, h\right)\left[\phi\left(\boldsymbol{r}^{\prime}\right)-\boldsymbol{p}^{T}\left(\boldsymbol{r}^{\prime}\right) \boldsymbol{\alpha}(\boldsymbol{r})\right]^{2} d \Omega_{\boldsymbol{r}}
$$

After minimization, we can write

$$
\widehat{\phi}(\boldsymbol{r})=\sum_{j=1}^{m} p_{j}(\boldsymbol{r}) \boldsymbol{\alpha}_{j}(\boldsymbol{r})=\sum_{j=1}^{n_{i}} N_{j}(\boldsymbol{r}) \phi_{j}
$$


where the associated shape functions, gathered in vector $N=\left(N_{1}, N_{2}, \ldots, N_{n_{i}}\right) \in \mathbb{R}^{n_{i}}$, are computed as

$$
\boldsymbol{N}^{T}(\boldsymbol{r})=\boldsymbol{p}^{T}(\boldsymbol{r}) \boldsymbol{M}^{-1}(\boldsymbol{r}) \boldsymbol{P}(\boldsymbol{r}) \boldsymbol{W}(\boldsymbol{r})
$$

where $\boldsymbol{P}$ is a $m \times n_{i}$ matrix where the basis functions are evaluated at each point of the stencil, namely $\boldsymbol{P}=\left[\boldsymbol{p}^{T}\left(\boldsymbol{r}_{j}\right)\right]_{j}$ and $\boldsymbol{M}(\boldsymbol{r})$ is the $m \times m$ moment matrix given by

$$
\boldsymbol{M}(\boldsymbol{r})=\boldsymbol{P}(\boldsymbol{r}) \boldsymbol{W}(\boldsymbol{r}) \boldsymbol{P}^{T}(\boldsymbol{r}) .
$$

Note that in Eq. (7), $n_{i} \neq m$. In practice, $n_{i}>m$ for a better conditioning of the moment matrix.

Diagonal matrix $\boldsymbol{W}(\boldsymbol{r})$ is derived from a kernel function evaluated at $\boldsymbol{r}_{j}-\boldsymbol{r}_{i}$ for the $n_{i}$ neighboring particles, as explained in the next section.

In order to improve the conditioning, the polynomial basis is locally defined and scaled: if the shape functions are evaluated at $\boldsymbol{r}_{i}$, the polynomial basis is evaluated at $\left(\boldsymbol{r}-\boldsymbol{r}_{i}\right) / h_{i}$. Thus, shape functions evaluated at $\boldsymbol{r}_{i}$ read:

$$
\boldsymbol{N}^{T}\left(\boldsymbol{r}_{i}\right)=\boldsymbol{p}^{T}(\boldsymbol{0}) \boldsymbol{M}^{-1}\left(\boldsymbol{r}_{i}\right) \boldsymbol{P}_{\Omega_{r_{i}}} \boldsymbol{W}\left(\boldsymbol{r}_{i}\right)=\boldsymbol{p}^{T}(\boldsymbol{0}) \boldsymbol{C}\left(\boldsymbol{r}_{i}\right)
$$

where matrix $\boldsymbol{C}\left(\boldsymbol{r}_{i}\right)$ is defined as

$$
\boldsymbol{C}(\boldsymbol{r})=\boldsymbol{M}^{-1}(\boldsymbol{r}) \boldsymbol{P}_{\Omega_{r}} \boldsymbol{W}(\boldsymbol{r})
$$

Once defined the MLS approximation in Eq. (7), the derivatives can be approximated as

$$
\nabla \widehat{\phi}(\boldsymbol{x})=\sum_{j=1}^{n_{i}} \phi_{j} \nabla N_{j}(\boldsymbol{x})
$$

We refer the reader to [17,32,33] for a complete description of the computation of MLS shape functions and derivatives.

\subsection{Practical notes about the kernel function}

The kernel function $\left(W_{i j}=W\left(\left\|\boldsymbol{r}_{i}-\boldsymbol{r}_{j}\right\|, h\right)\right)$ plays an important role in the formulation. It is used to weight the different particles that contribute to the approximation. In this work we use the cubic spline kernel [34], although many other possibilities do exist in the literature [35,36]

$$
W_{i j}=\beta_{D}\left\{\begin{array}{lr}
1-\frac{3}{2} q_{i j}^{2}+\frac{3}{4} q_{i j}^{3} & q_{i j} \leq 1, \\
\frac{1}{4}\left(2-q_{i j}\right)^{3} & 1<q_{i j} \leq 2, \\
0 & q_{i j} \leq 2,
\end{array}\right.
$$

where $q_{i j}$ is defined as $q_{i j}=\left\|\boldsymbol{r}_{j}-\boldsymbol{r}_{i}\right\| / h_{i}$, the constant $\beta_{D}=\frac{10}{7 \pi h^{2}}$ in two dimensional space and $\beta_{D}=\frac{1}{\pi h^{3}}$.

The smoothing length associated to the particle $i$ is variable for each particle and is computed as

$$
h_{i}=\sigma V_{i}^{\frac{1}{D}}
$$

where $D$ is the space dimensions number and $\sigma$ is a constant parameter. The number of neighbors is variable, depending on the value of $\sigma$ we shall set to $\sigma=1.5$ in the numerical applications. Note that this is different to the usual value in SPH-kernel approaches, which usually is $\sigma=2$. This implies that the number of particles involved in the approximation is decreased. In $2 \mathrm{D}$, this decrease is greater than $50 \%$, since the present formulation requires a number of 15-20 neighbor particles whereas kernel formulations with $\sigma=2$ involves around 50 particles for the computations.

\section{MLS based arbitrary Lagrangian-Eulerian meshless method}

The MLS shape functions verify the partition of unity property

$$
\sum_{j=1}^{n_{i}} N_{j}=1
$$


And, if the polynomial basis, $\boldsymbol{p}^{T}$ in Eq. (5), is at least linear, the shape functions also verify

$$
\sum_{j=1}^{n_{i}} \nabla N_{j}=0
$$

In the following, we also assume that for any smooth function $f(\boldsymbol{r})$, we can apply a one-point quadrature approximation at the particle $i$

$$
\int_{\Omega} f\left(\boldsymbol{r}_{i}\right) N\left(\boldsymbol{r}-\boldsymbol{r}_{i}\right) d \Omega \approx V_{i} f_{i} .
$$

where $V_{i}$ is the volume associated to particle $i$, and $\Omega$ is the volume of the compact support of the particle $i$. Therefore,

$$
\int_{\Omega} N\left(\boldsymbol{r}-\boldsymbol{r}_{i}\right) d \Omega \approx V_{i}
$$

In order to develop the formulation for the LEE equations, we start multiplying Eq. (1) by the MLS shape function $N_{i}=N\left(\boldsymbol{r}-\boldsymbol{r}_{i}, h_{i}\right)$ centered at particle $i$. Then we take the volumetric integral over the domain $\Omega$,

$$
\int_{\Omega} N_{i} L_{w} U d \Omega+\int_{\Omega} N_{i} \nabla \cdot \mathcal{F} d \Omega=0
$$

The divergence of the flux can be approximated using MLS as

$$
\int_{\Omega} N_{i} L_{w} \boldsymbol{U} d \Omega+\int_{\Omega} N_{i} \sum_{j=1}^{n}(\mathcal{F})_{j} \cdot \nabla N_{j} d \Omega=0
$$

Using a one point quadrature approximation Eq. (17), Eq. (20) reads

$$
V_{i} L_{w} \boldsymbol{U}_{i}+\int_{\Omega} N_{i} \sum_{j=1}^{n_{i}}(\mathcal{F})_{j} \cdot \nabla N_{j} d \Omega=0
$$

assuming that $(\mathcal{F})_{j}$ are point values,

$$
V_{i} L_{w} \boldsymbol{U}_{i}+\sum_{j=1}^{n_{i}}(\mathcal{F})_{j} \int_{\Omega} N_{i} \cdot \nabla N_{j} d \Omega=0
$$

Integrating by parts and applying the divergence theorem, we obtain

$$
V_{i} L_{w} \boldsymbol{U}_{i}+\sum_{j=1}^{n_{i}}(\mathcal{F})_{j}\left(\int_{\partial \Omega} N_{i} N_{j} \cdot \boldsymbol{n} d \Gamma-\int_{\Omega} N_{j} \cdot \nabla N_{i} d \Omega\right)=0
$$

where $\boldsymbol{n}$ is the unitary surface normal and $\partial \Omega$ is the surface of the volume $\Omega$.

If we add Eqs. (21) and (23) we obtain

$$
2 V_{i} L_{w} \boldsymbol{U}_{i}+\sum_{j=1}^{n_{i}}(\mathcal{F})_{j}\left(\int_{\partial \Omega} N_{i} N_{j} \cdot \boldsymbol{n} d \Gamma-\int_{\Omega} N_{j} \cdot \nabla N_{i} d \Omega+\int_{\Omega} N_{i} \cdot \nabla N_{j} d \Omega\right)=0
$$

Eq. (24) reads

$$
V_{i} L_{\boldsymbol{w}} \boldsymbol{U}_{i}+\frac{1}{2} \sum_{j=1}^{n_{i}}\left(\mathcal{F}_{j}\right)\left(\int_{\partial \Omega} N_{i} N_{j} \cdot \boldsymbol{n} d \Gamma-\int_{\Omega} N_{j} \cdot \nabla N_{i} d \Omega+\int_{\Omega} N_{i} \cdot \nabla N_{j} d \Omega\right)=0
$$

Now, we add and subtract $\mathcal{F}_{i}$ to the fluxes. Note that this operation has no effect at the continuum level, since, using the properties of the MLS shape functions Eq. (16)

$$
\sum_{j=1}^{n_{i}}(\mathcal{F})_{i} \int_{\Omega} N_{i} \nabla N_{j} d \Omega=(\mathcal{F})_{i} \int_{\Omega} N_{i} \sum_{j=1}^{n_{i}} \nabla N_{j} d \Omega=0
$$


and using a one-point quadrature for the volume integrals we arrive to

$$
V_{i} L_{\boldsymbol{w}} \boldsymbol{U}_{i}+\sum_{j=1}^{n_{i}}\left[\frac{1}{2}\left(\mathcal{F}_{j}+\mathcal{F}_{i}\right)-\mathcal{F}_{i}\right]\left(\int_{\partial \Omega} N_{i} N_{j} \cdot \boldsymbol{n} d \Gamma-V_{j} \nabla N_{i j}+V_{i} \nabla N_{j i}\right)=0
$$

where $N_{i j}=N\left(\boldsymbol{r}_{j}-\boldsymbol{r}_{i}, h_{i}\right)$ and $N_{j i}=N\left(\boldsymbol{r}_{i}-\boldsymbol{r}_{j}, h_{j}\right)$. As it is customary in standard SPH methodology let us assume there is no rigid boundary, so the boundary integral is equal to zero. The case where boundaries are present is addressed in Section 4.2.

$$
V_{i} L_{w} U_{i}+\sum_{j=1}^{n_{i}}\left[\frac{1}{2}\left(\mathcal{F}_{j}+\mathcal{F}_{i}\right)-\mathcal{F}_{i}\right]\left(-V_{j} \nabla N_{i j}+V_{i} \nabla N_{j i}\right)=0
$$

Now, we define the numerical flux as $\boldsymbol{G}_{i j} \approx \frac{1}{2}\left(\mathcal{F}_{j}+\mathcal{F}_{i}\right)$ (the difference in the two terms is the numerical dissipation).

$$
V_{i} L_{w} \boldsymbol{U}_{i}+\sum_{j=1}^{n_{i}}\left[\boldsymbol{G}_{i j}-\mathcal{F}_{i}\right]\left(-V_{j} \nabla N_{i j}+V_{i} \nabla N_{j i}\right)=0
$$

Therefore, the system of conservation laws can be discretized as

$$
\frac{d\left(V_{i} \boldsymbol{U}_{i}\right)}{d t}+\sum_{j=1}^{n_{i}}\left[\boldsymbol{G}_{i j}-\mathcal{F}_{i}\right]\left(-V_{j} \nabla N_{i j}+V_{i} \nabla N_{j i}\right)=0
$$

If an Eulerian configuration is chosen for a set of particles distributed with equal weights $V_{i}$, Eq. (30) is very close to that obtained in [11] using the Finite Point Method (FPM) for the discretization of the LEE. However, there are some differences, such as the symmetrization of the gradient of the shape functions, which is not performed in the FPM.

It is also interesting to remark that, if we use a kernel approximation instead of MLS shape functions we arrive to

$$
\frac{d\left(V_{i} \boldsymbol{U}_{i}\right)}{d t}=-\sum_{j=1}^{n_{i}} V_{i} V_{j} 2\left(\boldsymbol{G}_{i j}-\mathcal{F}_{i}\right) \cdot \nabla W_{i j}
$$

due to the anti-symmetry property of the kernel gradient i.e.: $\nabla W_{i j}=-\nabla W_{j i}$. This is the SPH-ALE formulation presented in [18] in the form proposed in [19]. We note that using the presented formulation, the origin and the necessity of the term $\mathcal{F}_{i}$ is clearly explained. In this work $\boldsymbol{G}_{i j}$ is computed at point $\boldsymbol{r}_{i j}$ using the Rusanov numerical flux [37].

$$
\boldsymbol{G}_{i j}=\frac{1}{2}\left(\boldsymbol{H}_{i j}^{+}+\boldsymbol{H}_{i j}^{-}\right) \cdot \boldsymbol{n}-\frac{1}{2} S_{i j}^{+} \Delta \boldsymbol{U}_{i j} \cdot \boldsymbol{n},
$$

where $S_{i j}^{+}$is the maximum eigenvalue of the Jacobian matrix which writes in the ALE framework in terms of the left and right sounds speeds $\left(c_{i j}^{ \pm}\right)$as

$$
S_{i j}^{+}=\max \left(\left(\boldsymbol{u}_{0}-\boldsymbol{w}\right) \cdot \boldsymbol{n}+c_{i j},\left(\boldsymbol{u}_{0}-\boldsymbol{w}\right) \cdot \boldsymbol{n}-c_{i j}\right),
$$

Quantities $\boldsymbol{H}_{i j}^{-}$and $\boldsymbol{H}_{i j}^{+}$denote the flux approximations of $\boldsymbol{H}$ on the left and right sides of the integration point $\boldsymbol{r}_{i j}$ with the positive orientation given by $\boldsymbol{r}_{j}-\boldsymbol{r}_{i}$ and represented by the normalized vector $\boldsymbol{n}_{i j}$. The jump of the conservative vector is defined as $\Delta \boldsymbol{U}_{i j}=\boldsymbol{U}_{i j}^{+}-\boldsymbol{U}_{i j}^{-}$.

It is important to remark that since the number of neighboring particles inside the compact support using this formulation decreases compared with that of the SPH-ALE method, the number of Riemann problems to be computed is also decreased, which is an important advantage from a computational point of view.

The equation of the particles motion reads as

$$
\frac{d \boldsymbol{r}_{i}}{d t}=\sum_{j=1}^{n_{i}} N_{i j} \boldsymbol{w}_{j}
$$

As in the traditional SPH, the equation of evolution of the particles volumes follows a similar rationale. For a generic function $f(\boldsymbol{x}, t)$ it is known that

$$
\frac{d}{d t} \int_{\Omega} f d \Omega=\int_{\Omega} \frac{\partial f}{\partial t} d \Omega+\int_{\partial \Omega} f \boldsymbol{u} \cdot \boldsymbol{n} d \Gamma
$$


If we consider the constant function, $f(x, t)=1$, the volume $\Omega$ equal to $V_{i}$ and the velocity at the boundary equal to $\boldsymbol{w}$ we obtain

$$
\frac{d}{d t} \int_{V_{i}} d \Omega=\int_{\partial V_{i}} \boldsymbol{w} \cdot \boldsymbol{n} d \Gamma=\int_{V_{i}} \nabla \cdot \boldsymbol{w} d \Omega
$$

Introducing the MLS approximation,

$$
\frac{d}{d t} \int_{V_{I}} d \Omega=\sum_{j=1}^{n_{i}} \int_{V_{I}} \boldsymbol{w}_{j} \nabla \cdot N_{j i} d \Omega
$$

Following the same procedure as in the system of conservation laws we obtain

$$
\frac{d V_{i}}{d t}=\sum_{j=1}^{n_{i}}\left[\boldsymbol{w}_{i j}-\boldsymbol{w}_{i}\right]\left(-V_{j} \nabla N_{i}+V_{i} \nabla N_{j i}\right)
$$

It is important to remark that the numerical scheme is formally second-order, even though the reconstruction of Riemann states is higher-order [25].

\subsection{High-order reconstruction of the Riemann states}

In order to increase the accuracy of the method and to obtain a numerical method suitable for applications in CAA, we introduce high-order reconstructions of the Riemann states $\left(\boldsymbol{U}_{i j}^{+}\right.$and $\left.\boldsymbol{U}_{i j}^{-}\right)$used for the computation of $\boldsymbol{H}_{i j}^{+}$and $\boldsymbol{H}_{i j}^{-}$in Eq. (32). This is achieved using Taylor polynomials centered at the particles. As an example, the quadratic reconstruction associated to particle $i$ and evaluated at $\boldsymbol{r}_{i j}$ reads

$$
\boldsymbol{U}_{i j}^{+}=\boldsymbol{U}_{i}+\nabla \boldsymbol{U}_{i} \cdot\left(\boldsymbol{r}_{i j}-\boldsymbol{r}_{i}\right)+\frac{1}{2}\left(\boldsymbol{r}_{i j}-\boldsymbol{r}_{i}\right)^{T} \nabla^{2} \boldsymbol{U}_{i}\left(\boldsymbol{r}_{i j}-\boldsymbol{r}_{i}\right),
$$

The gradient $\boldsymbol{\nabla} \boldsymbol{U}_{i}$, the Hessian matrix $\nabla^{2} \boldsymbol{U}_{i}$ and successive derivatives required are computed using MLS approximations $[16,17,20]$. Note that the use of MLS does not introduce any increase of computational cost, since the MLS shape functions are already computed for the integration procedure.

\subsection{Solid wall boundaries}

For particle $i$ with a compact support that crosses a boundary, the assumption of zero boundary integral no longer holds. In this case, the boundary integral must be computed as follows,

We recall Eq. (27) which reads

$$
V_{i} L_{w} \boldsymbol{U}_{i}+\sum_{j=1}^{n_{i}}\left[\frac{1}{2}\left(\mathcal{F}_{j}+\mathcal{F}_{i}\right)-\mathcal{F}_{i}\right]\left(\int_{\partial \Omega} N_{i} N_{j} \cdot \boldsymbol{n} d \Gamma-V_{j} \nabla N_{i j}+V_{i} \nabla N_{j i}\right)=0
$$

For non-interior particles the boundary term cannot be neglected. Following [38]

$$
\int_{\partial \Omega} N_{i} N_{j} \cdot \boldsymbol{n} d \Gamma=\int_{\Omega} N_{i} \nabla N_{j} d \Omega+\int_{\Omega} N_{j} \nabla N_{i} d \Omega
$$

which leads to

$$
V_{i} L_{w} \boldsymbol{U}_{i}+\sum_{j=1}^{n_{i}}\left[\frac{1}{2}\left(\mathcal{F}_{j}+\mathcal{F}_{i}\right)-\mathcal{F}_{i}\right]\left(2 V_{i} \nabla N_{j i}\right)=0
$$

\subsection{Numerical methodology}

Finally, for the sake of clarity, the system of equations to be solved is summarized. Recalling Eqs. (14), (30), (34) and (44), the system of conservation laws is discretized as

$$
\frac{d\left(V_{i} \boldsymbol{U}_{i}\right)}{d t}+\sum_{j=1}^{n_{i}}\left[\boldsymbol{G}_{i j}-\mathcal{F}_{i}\right]\left(-V_{j} \nabla N_{i j}+V_{i} \nabla N_{j i}\right)=0
$$

for the case of non-interior particles, Eq. (41) is used instead. 

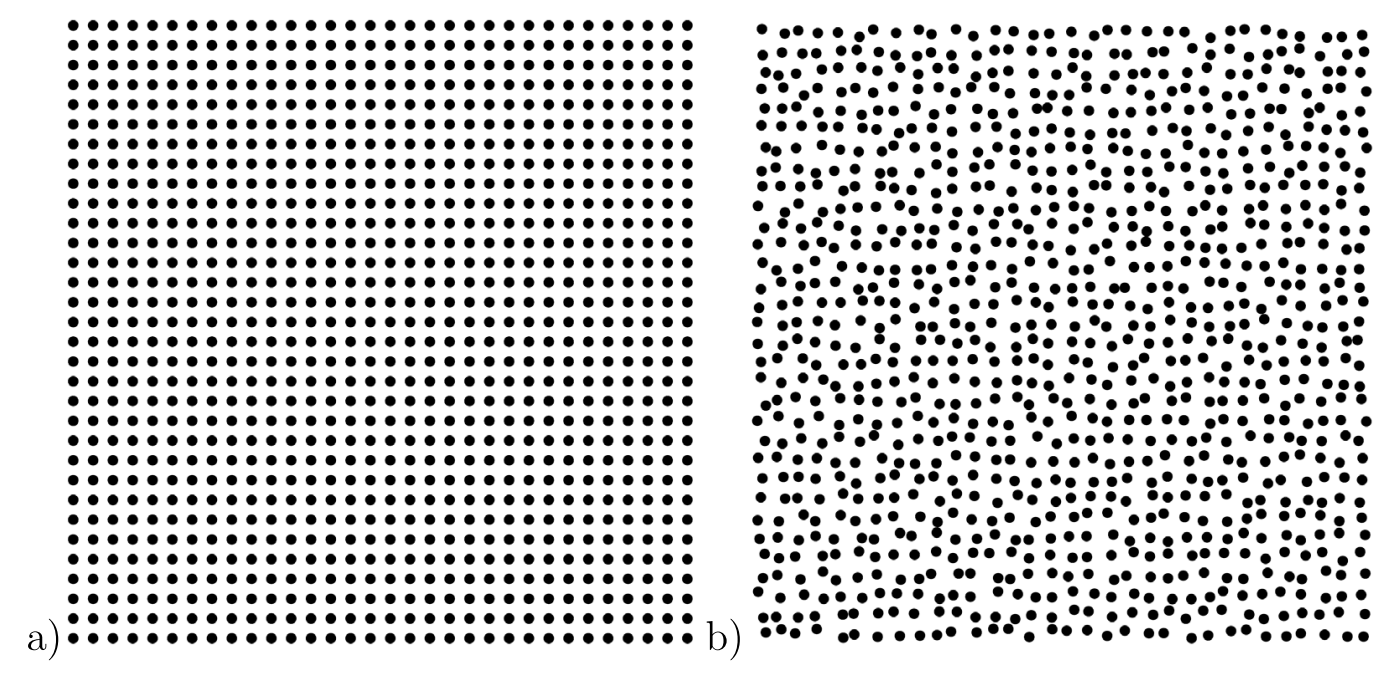

Fig. 2. Particle distributions used for $2 D$ Reconstruction test case. Equidistant (a) and random (b) distributions of 1024 particles.

The particle position is computed as

$$
\frac{d \boldsymbol{r}_{i}}{d t}=\sum_{j=1}^{n_{i}} N_{i j} \boldsymbol{w}_{j}
$$

The evolution of the particle volume reads

$$
\frac{d V_{i}}{d t}=\sum_{j=1}^{n_{i}}\left[\boldsymbol{w}_{i j}-\boldsymbol{w}_{i}\right]\left(-V_{j} \nabla N_{i}+V_{i} \nabla N_{j i}\right)
$$

And finally, the smoothing length is updated as

$$
h_{i}=\sigma V_{i}^{\frac{1}{D}}
$$

In this work we have used an explicit fourth-order Runge-Kutta time integration scheme.

\section{Numerical results}

This section presents the numerical results for several benchmark problems aimed at assessing the accuracy and efficiency of the proposed method for CAA problems. The test cases addressed here are listed as

$2 D$ pure reconstruction test. This case is devoted to study the accuracy of the variables reconstruction.

Gaussian acoustic pulse. This case is commonly used in the literature as a first test of the accuracy of the schemes for CAA.

Acoustic pulse reflection and duct acoustic propagation. These benchmarks are devoted to test the accuracy of the proposed numerical method when solid walls are present in the domain, and wave reflections occur.

Monopole source radiation in uniform mean flow. This test case examines the ability of the proposed scheme to simulate long-time propagation of noise. Moreover, it is also a test for the absorbing boundary conditions.

Acoustic wave scattering by a circular cylinder. This test case is devoted to analyze the behavior of the numerical method with curved boundary walls.

All the cases are performed using a cubic reconstruction of the Riemann states, except when it is explicitly mentioned.

\subsection{D pure reconstruction test}

In order to demonstrate the accuracy and the formal order of reconstruction of the variables, we perform a reconstruction test case as described in [20]. The $2 \mathrm{D}$ square domain $\Omega=[-1.5,1.5] \times[-1.5,1.5]$ is discretized 
Table 1

Accuracy orders $O_{2}^{N}, O_{\infty}^{N}$ and $L_{2}^{N}, L_{\infty}^{N}$ norms of the reconstruction error for the 2D Reconstruction test cases using a cubic MLS reconstruction.

\begin{tabular}{|c|c|c|c|c|c|}
\hline & $N$ & $L_{2}^{N}$ error & $O_{2}^{N}$ & $L_{\infty}^{N}$ error & $O_{\infty}^{N}$ \\
\hline \multirow{6}{*}{ 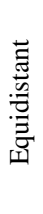 } & 1,024 & $6.62 \times 10^{-5}$ & - & $3.34 \times 10^{-4}$ & - \\
\hline & 2,304 & $1.40 \times 10^{-5}$ & 3.825 & $7.36 \times 10^{-5}$ & 3.741 \\
\hline & 4,096 & $4.55 \times 10^{-6}$ & 3.916 & $2.41 \times 10^{-5}$ & 3.873 \\
\hline & 9,216 & $9.13 \times 10^{-7}$ & 3.959 & $4.90 \times 10^{-6}$ & 3.936 \\
\hline & 16,384 & $2.91 \times 10^{-7}$ & 3.981 & $1.56 \times 10^{-6}$ & 3.968 \\
\hline & 36,864 & $5.76 \times 10^{-8}$ & 3.991 & $3.11 \times 10^{-7}$ & 3.984 \\
\hline \multirow{6}{*}{ 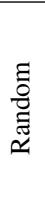 } & 1,024 & $9.30 \times 10^{-5}$ & - & $5.94 \times 10^{-4}$ & - \\
\hline & 2,304 & $2.01 \times 10^{-5}$ & 3.772 & $1.58 \times 10^{-4}$ & 3.271 \\
\hline & 4,096 & $6.58 \times 10^{-6}$ & 3.889 & $5.59 \times 10^{-5}$ & 3.608 \\
\hline & 9,216 & $1.30 \times 10^{-6}$ & 3.990 & $1.16 \times 10^{-5}$ & 3.869 \\
\hline & 16,384 & $4.19 \times 10^{-7}$ & 3.945 & $3.88 \times 10^{-6}$ & 3.821 \\
\hline & 36,864 & $8.35 \times 10^{-8}$ & 3.982 & $7.82 \times 10^{-7}$ & 3.950 \\
\hline
\end{tabular}

a)

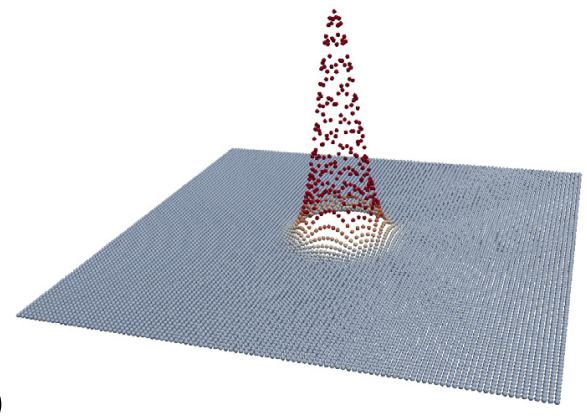

b)
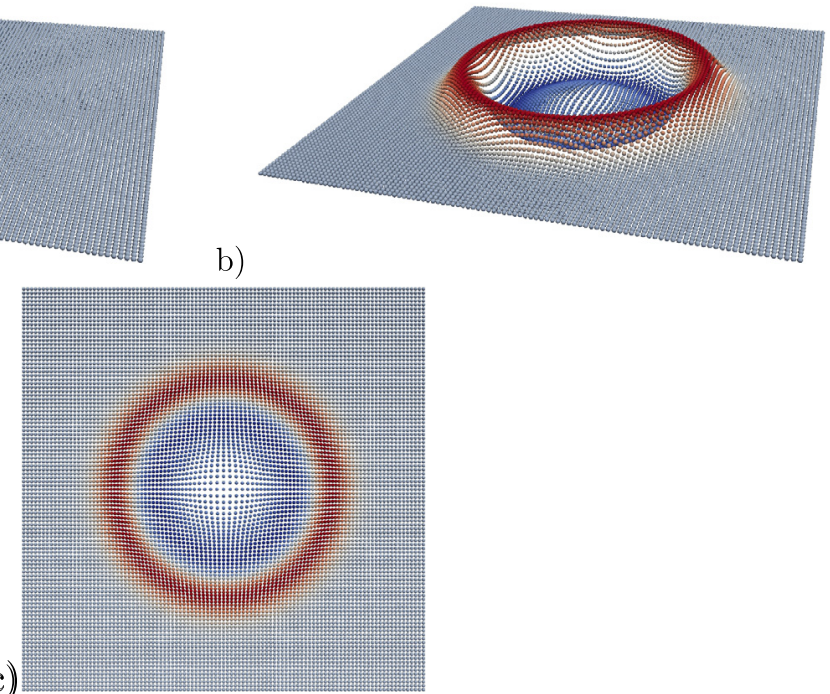

Fig. 3. Propagation of an acoustic pulse. Density fluctuations field. Initial fluctuations (a), solution at $t=10$ (b) and (c) obtained with the proposed scheme.

with equidistant and randomly particle distributions, as shown in Fig. 2. In this example we define the function to be reconstructed as

$$
f(\boldsymbol{r})=a e^{-\frac{|r|^{2}}{2 b}}
$$

where $\boldsymbol{r}$ is the position of the particle, $a=1 /(\sqrt{2 b \pi})$ and $b=0.20$. First, we set the know value of the function to each particle. Next, MLS derivatives are computed at the position of each particle and Taylor reconstruction is performed in order to compute the value at the midpoint between particles.

$L_{2}^{N}$ and $L_{\infty}^{, N}$ norms of the reconstruction error are computed for each distribution with $N$ particles. Convergence rates are obtained by using the errors obtained for two different sets with $N_{1}$ and $N_{2}$ particles by using the formula

$$
O^{N}=\frac{\log \left(L^{N_{1}} / L^{N_{2}}\right)}{\log \left(\sqrt{N_{2} / N_{1}}\right)} .
$$



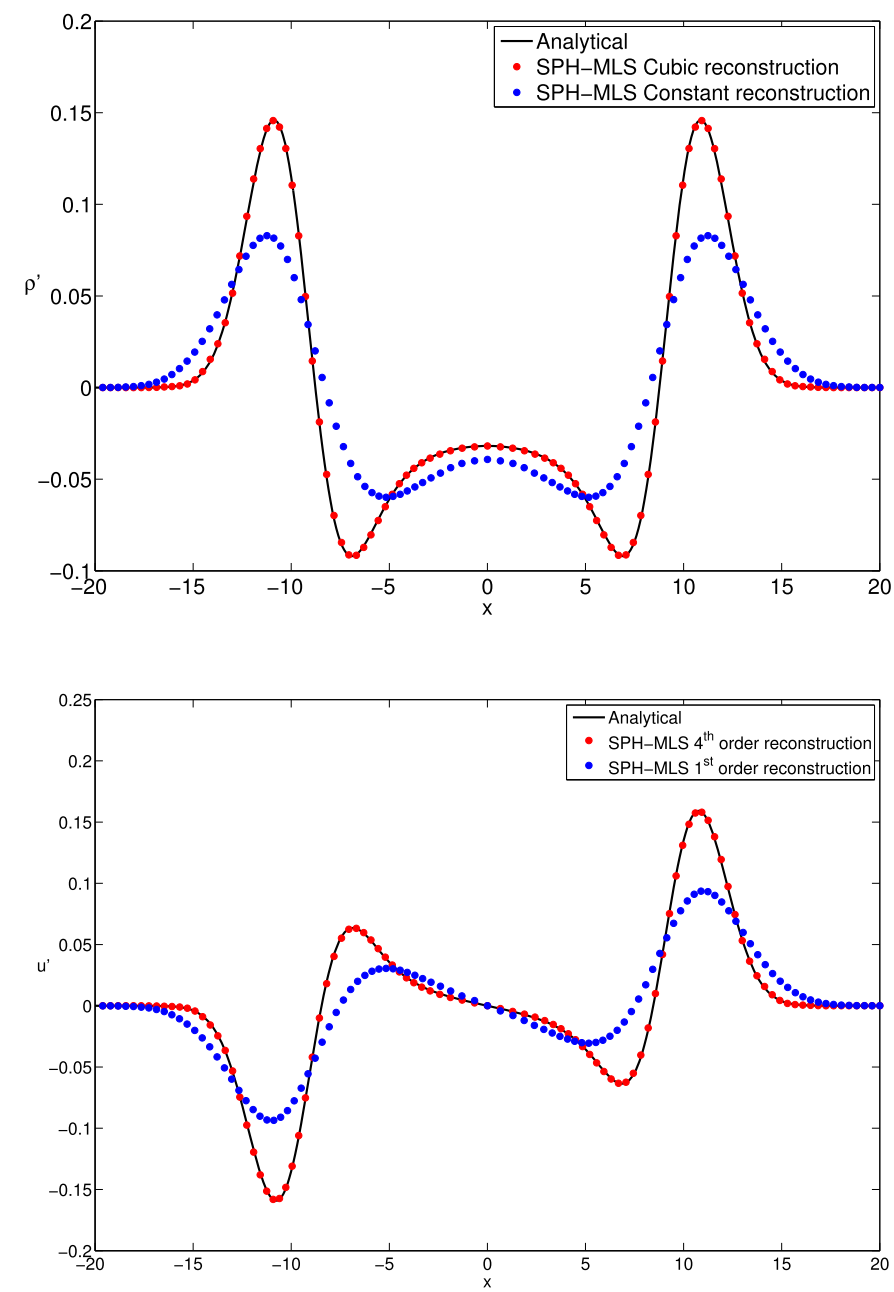

Fig. 4. Propagation of an acoustic pulse. Density (top), and $u$-velocity (bottom) perturbations at $t=10$ obtained with the proposed scheme using both, a constant reconstruction and a cubic reconstruction of the Riemann states.

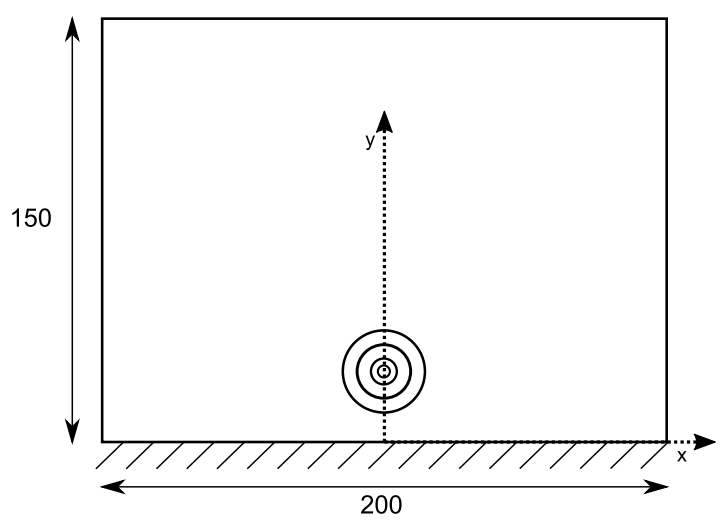

Fig. 5. Acoustic pulse reflection with zero mean flow. Schematic setup of the problem. 

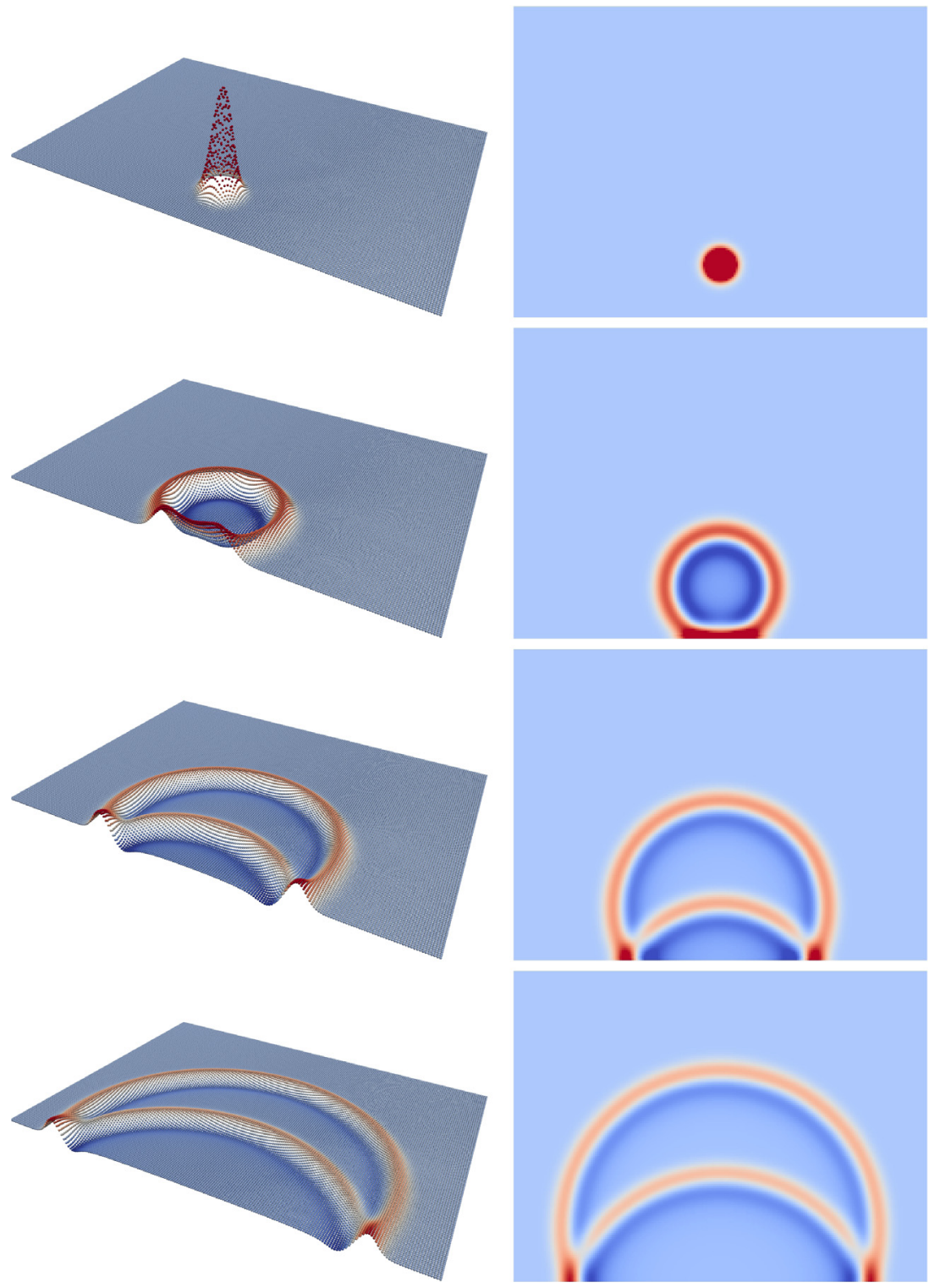

Fig. 6. Acoustic pulse reflection with no convection. Density fluctuations results for $t=0, t=25, t=50, t=75$, using [201 $\times 151]$ particles.

Errors and convergence rates for cubic reconstruction are reported in Table 1 for equidistant and random distributions of particles. It is observed that the optimal orders of accuracy are recovered independently of the location of the particles.

\subsection{Gaussian acoustic pulse}

In this example the accuracy of the proposed methodology in the simulation of the propagation of a Gaussian acoustic pulse is tested. This case is commonly used in the literature as a first test of the accuracy of the numerical schemes [11,33]. The computational domain is a $\Omega=[-20,20] \times[-20,20]$ and it is discretized with an homogeneous distribution of $101 \times 101$ particles. The initial condition for the fluctuating variables is defined as

$$
\rho^{\prime}(x, y, t=0)=e^{-\kappa r^{2}}, \quad u^{\prime}=v^{\prime}=0, \quad p^{\prime}(x, y, t=0)=e^{-\kappa r^{2}}
$$




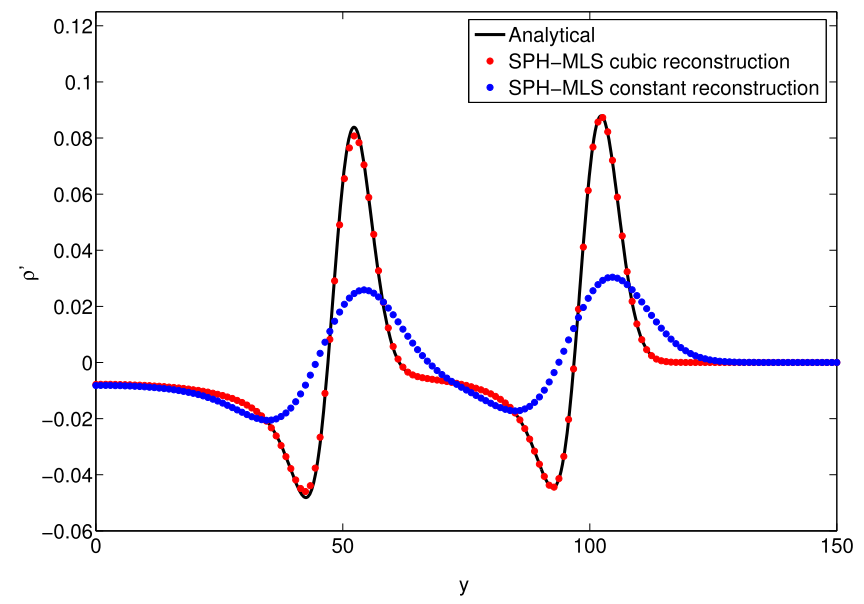

Fig. 7. Acoustic pulse reflection with no convection. Density fluctuations results for a cut along $x=x_{p}$. Comparison of the results using a linear and a cubic reconstruction using $[201 \times 151]$ particles.

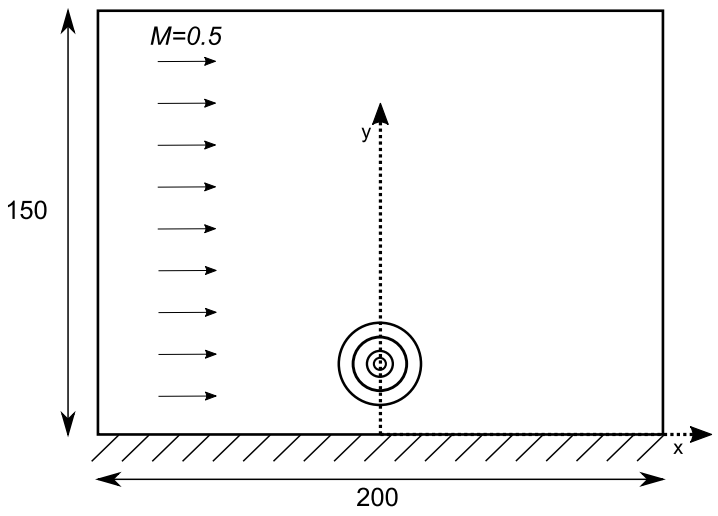

Fig. 8. Acoustic pulse reflection in uniform mean flow. Schematic setup of the problem.

where $\kappa=\ln (2) / b^{2}, b=2$ and $r$ indicates the distance to the initial location of the pulse. The mean velocity is set to zero.

The numerical solution is computed at $t=10$ using a Lagrangian approach. The Lagrangian frame velocity is defined as the fluctuation velocity of the particles.

In Fig. 3 we plot the initial condition and the final result for the density fluctuations for the cubic reconstructions of the Riemann states. The initial perturbation is propagated symmetrically as expected.

Moreover, in Fig. 4 we plot the results for the density and velocity fluctuations compared with the analytical solution [39]. The computed $L_{2}$ norm of the density fluctuations are $2.090 \times 10^{-2}$ for the constant reconstruction and $3.477 \times 10^{-4}$ for the cubic reconstruction. It is observed the great increase in accuracy using the high-order reconstruction, which reproduces very accurately the analytic solution.

\subsection{Acoustic pulse reflection}

This set of tests is performed in order to test the accuracy of the proposed method in presence of solid walls. These tests were proposed in the ICASE/LaRC workshop [40] as a benchmark for numerical methods for CAA. In this section we perform three numerical test with gradually increasing difficulty. In the first test case we study the interaction of a Gaussian acoustic pulse with a solid wall. The second test case addresses the same problem but under the action of a mean flow with Mach number $M=0.5$. The third test case of this section considers the propagation 

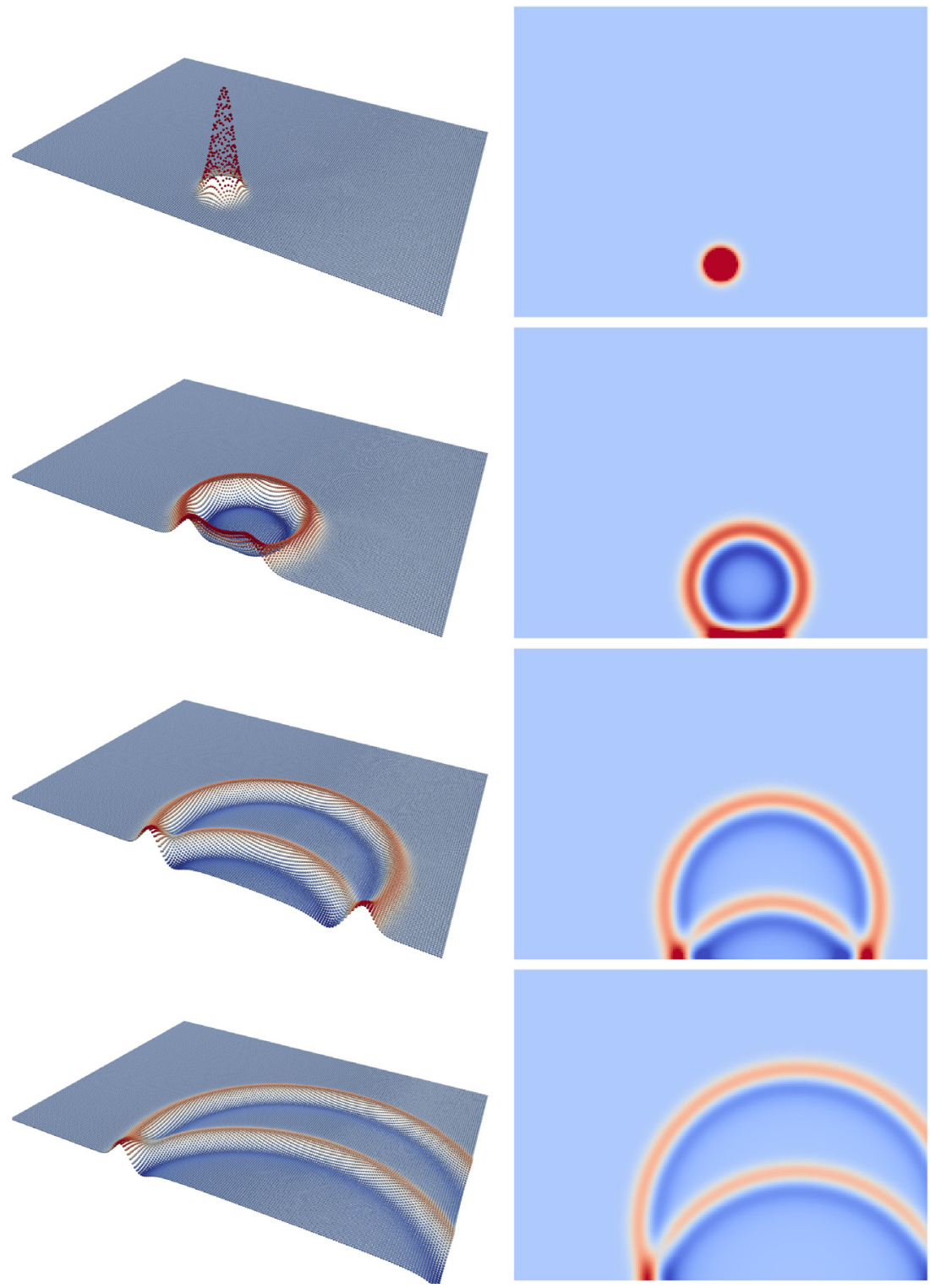

Fig. 9. Acoustic pulse reflection under the action of a mean $M=0.5$ flow. Density fluctuations results for $t=0, t=25, t=50, t=75$, using $[201 \times 151]$ particles.

of a Gaussian pulse placed in a duct under the action of a mean $M=0.5$ flow. Finally, a 3D extension of the first case is analyzed.

\subsubsection{D-acoustic pulse reflection: zero mean flow test case}

The schematic setup of the problem is indicated in Fig. 5.

We consider a Cartesian distribution of particles $201 \times 151$ and we solve the problem in an Eulerian framework. The initial position of the pulse is $\left(x_{p}, y_{p}\right)=(0,25)$, and it is defined as

$$
\rho^{\prime}(x, y, t=0)=e^{-\kappa r^{2}}, \quad u^{\prime}=v^{\prime}=0, \quad p^{\prime}(x, y, t=0)=e^{-\kappa r^{2}}
$$

with $\kappa=\ln (2) / b^{2}, b=5$ and $r$ indicates the distance to the initial location of the pulse. 


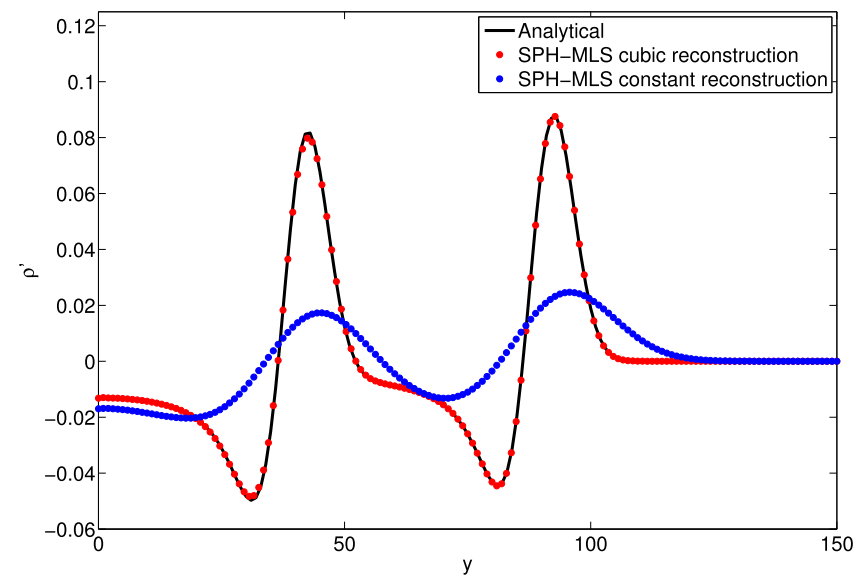

Fig. 10. Acoustic pulse reflection under the action of a mean $M=0.5$ flow. Density fluctuations results for a cut along $x=x_{p}$. Comparison of the results using a linear and a cubic reconstruction using $[201 \times 151]$ particles.

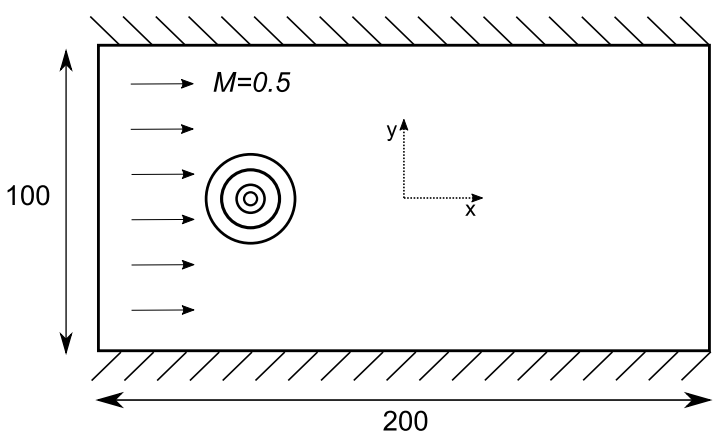

Fig. 11. Duct acoustic propagation. Setup of the problem.

In Fig. 6 we show the density fluctuations field for $t=0, t=25, t=50, t=75$. We observe that the wave is perfectly reflected by the wall, with no numerical artifacts in the solution.

In Fig. 7 we show the results at time $t=75$ for the no convection case. In particular, we show the density fluctuations in a cut along $x=x_{p}$. It is seen the perfect matching with the analytical solution, computed as described in [39]. It is also shown in the figure the result using a constant reconstruction in the Riemann problem. The results are clearly improved with the high-order reconstruction.

\subsection{2. $2 D$-acoustic pulse reflection: $M=0.5$ mean flow test case}

The schematic setup of the problem is indicated in Fig. 8.

The case with convection corresponds to the first problem of the Category 4 of the ICASE workshop [40]. The results are shown in Figs. 9 and 10. Again, the numerical results match perfectly the analytical solution, computed following [39].

\subsubsection{D-acoustic pulse reflection: duct acoustic propagation}

Next, we solve the propagation of a Gaussian pulse placed in a duct under the action of a mean $M=0.5$ flow. The setup of this problem is shown in Fig. 11 and is taken from [41].

The initial position of the pulse is $\left(x_{p}, y_{p}\right)=(-50,0)$, and it is defined as

$$
\rho^{\prime}(x, y, t=0)=e^{-\kappa r^{2}}, \quad u^{\prime}=v^{\prime}=0, \quad p^{\prime}(x, y, t=0)=e^{-\kappa r^{2}}
$$

with $\kappa=\ln (2) / b^{2}, b=6$ and $r$ indicates the distance to the initial location of the pulse. For this test case, we use a random distribution of 20,330 particles, as it is shown in Fig. 12. Note that the choice of this grid is made with 


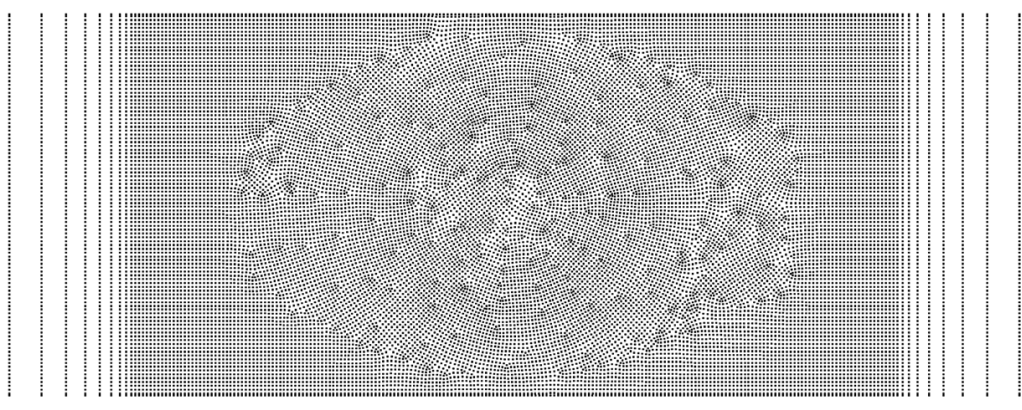

Fig. 12. Duct acoustic propagation. Distribution of the particles.
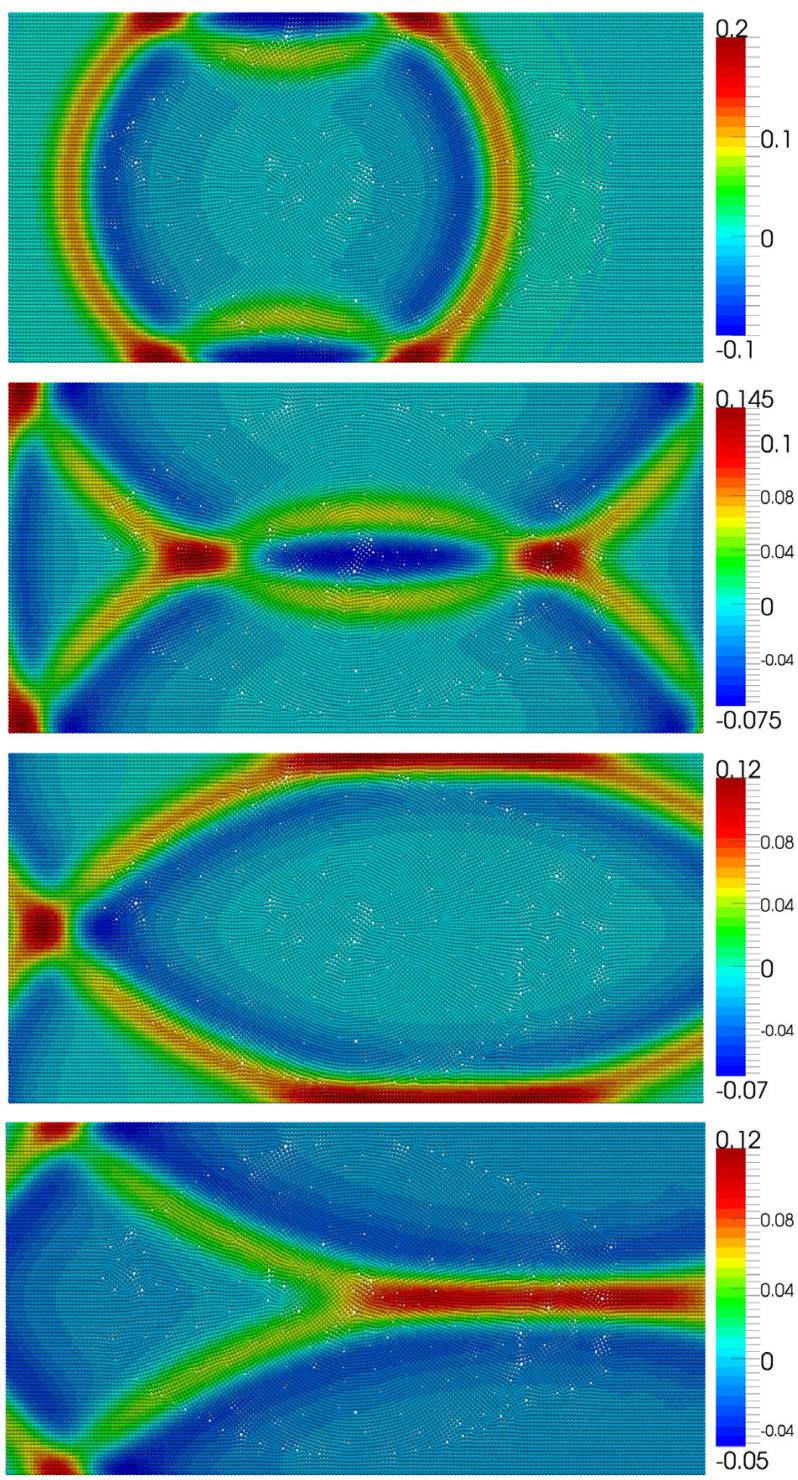

Fig. 13. Duct acoustic propagation. Pressure field at times $t=60, t=110, t=150$ and $t=300$. 

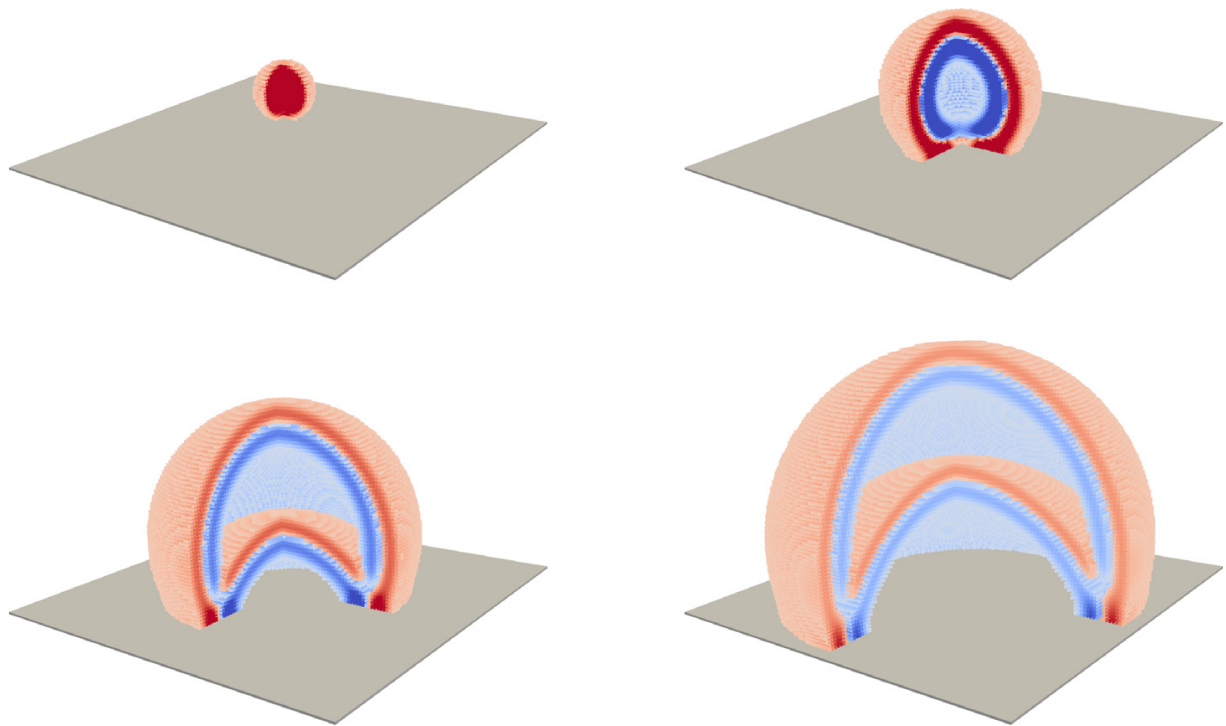

Fig. 14. 3D-Acoustic pulse reflection with no convection. Density fluctuations results for $t=0, t=25, t=50, t=75$, using [115 $\times 101 \times 151]$ particles.

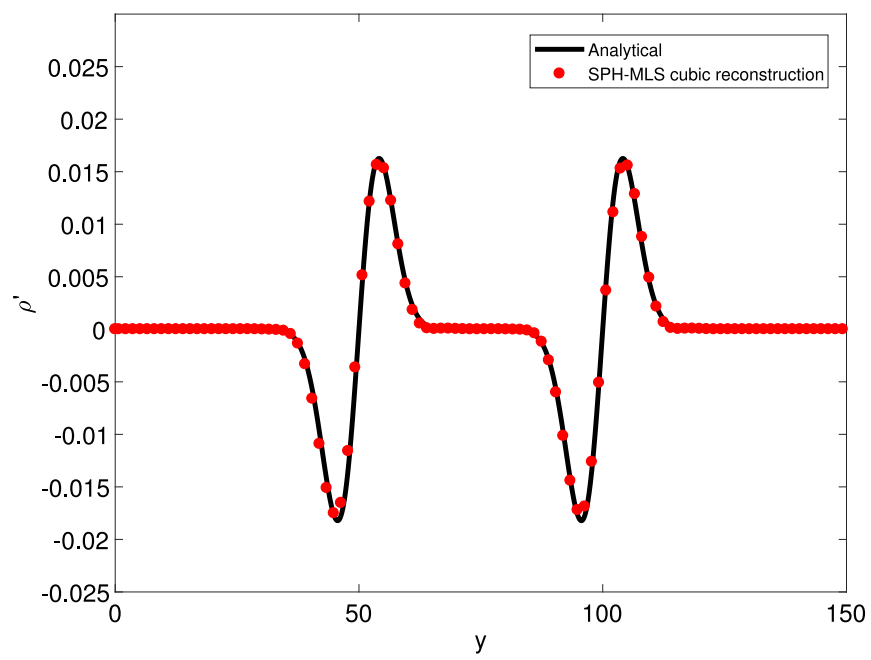

Fig. 15. 3D-Acoustic pulse reflection with no convection. Density fluctuations results for a cut along $x=x_{p}, z=z_{p}$.

the purpose of showing the ability of the method to obtain very accurate results with scattered distribution of data. However, it is not in general a recommended distribution for solving CAA problems.

In spite of this distribution, it is shown in Fig. 13 that the method obtains very accurate results, and that it is free of any spurious noise. The results could be compared with other methods proposed in the literature $[11,41,42]$.

\subsubsection{D-acoustic pulse reflection}

The 3D extension of the Acoustic pulse benchmark is devoted to test the accuracy of the proposed numerical method when solid walls are present on a 3D domain. The setup of the problem described in Fig. 5 is extruded in $z$-direction, and therefore the computational domain is $\Omega=[-100,0,-100] \times[100,150,100]$.

We consider a uniform distribution of particles $115 \times 101 \times 115$ and we solve the problem in an Eulerian framework. The initial position of the pulse is $\left(x_{p}, y_{p}, z p\right)=(0,25,0)$, and it is defined as

$$
\rho^{\prime}(x, y, z, t=0)=e^{-\kappa r^{2}}, u^{\prime}=v^{\prime}=w^{\prime}=0, p^{\prime}(x, y, z, t=0)=e^{-\kappa r^{2}}
$$




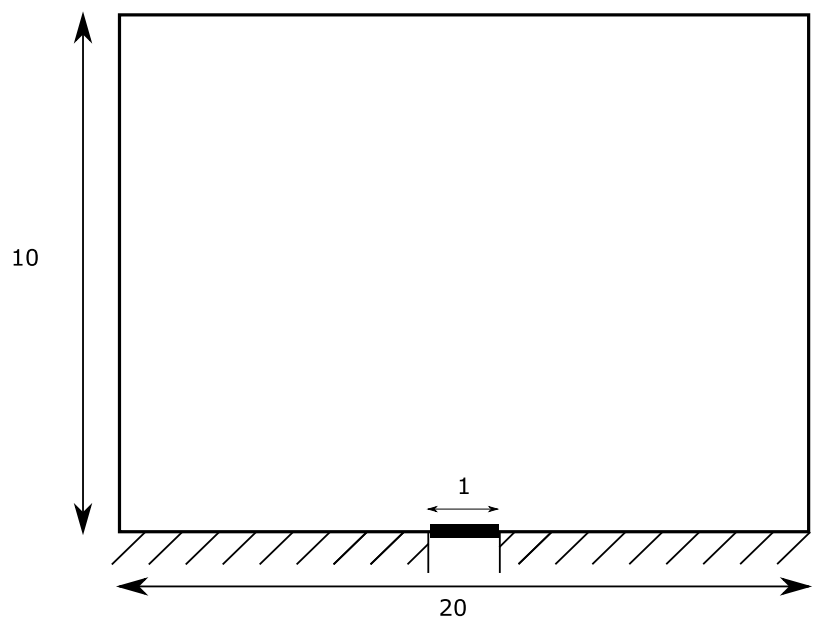

Fig. 16. Baffled Piston test case. Schematic setup of the problem.
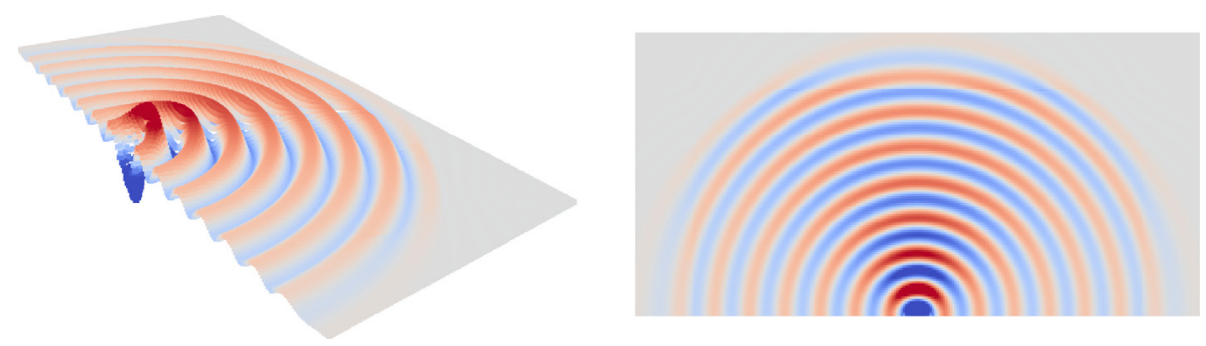

Fig. 17. Baffled Piston test case. Pressure fluctuations results at $t=12$.

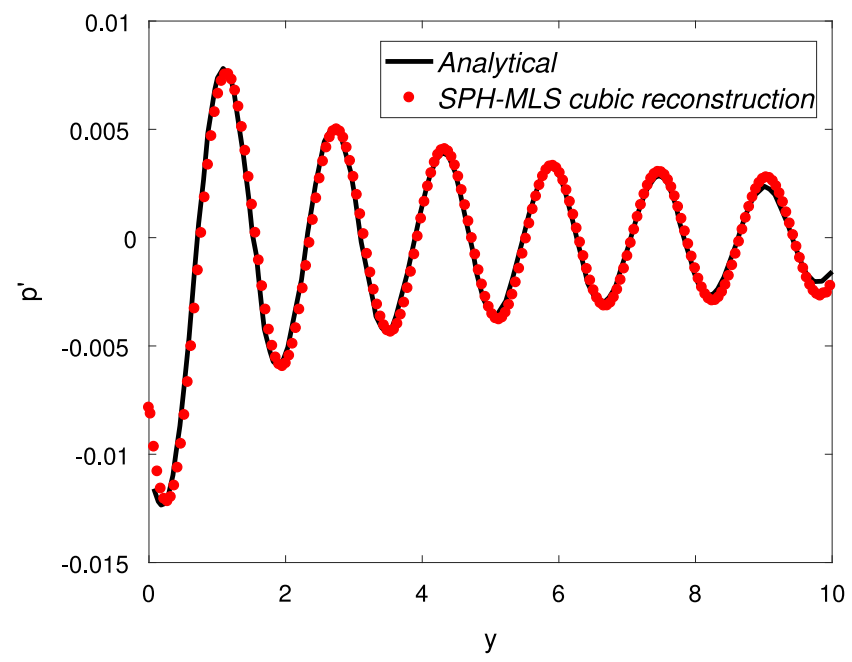

Fig. 18. Baffled Piston test case. Pressure fluctuations results for a cut along $x=0$ at $t=12$. 

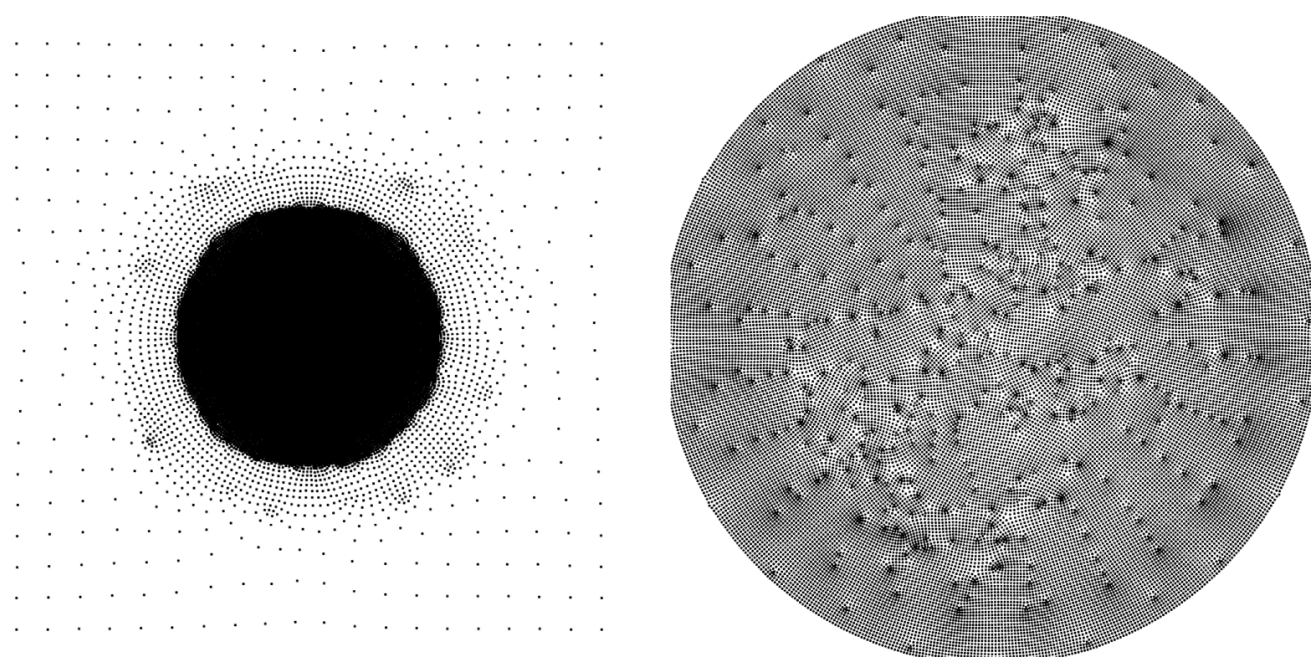

Fig. 19. Monopole source radiation in uniform $M=0.5$ mean flow. Particle distribution. On the left we show the whole domain including the absorbing layer, whereas on the right we shown the computational domain of interest.

with $\kappa=\ln (2) / b^{2}, b=5$ and $r$ indicates the distance to the initial location of the pulse. In Fig. 14 we show the density fluctuations field for $t=0, t=25, t=50, t=75$, whereas in Fig. 15 we show the result at time $t=75$. In particular, we show the density fluctuations in a cut along $x=x_{p}, z=z_{p}$. It is observed a good match with the analytical solution.

\subsection{Baffled Piston}

One of the advantages of the proposed approach is the easy handling of moving boundaries. In order to test the method in such situations we compute the acoustic wave propagation produced by an oscillating piston. This test case for Computational Aeroacoustics was presented in $[40,43]$. The schematic setup of this problem is represented in Fig. 16.

The solid wall boundary condition is imposed at the wall. The piston has a prescribed motion defined by

$$
v^{\prime}(x, y, t)=V_{0} \cos (\omega t)
$$

where the angular frequency of the piston is set to $\omega=4$ and the amplitude is $V_{0}=0.01$. The computational domain is discretized with $201 \times 101$ particles. The problem is solved using a Lagrangian framework until a final time $t=12$. The final acoustic pressure, represented in Fig. 17, is compared with the analytical solution and represented in Fig. 18. It is observed a good match with the analytical solution.

\subsection{Monopole source radiation in uniform $M=0.5$ mean flow}

This test case reproduces the radiation of an acoustic source in a uniform subsonic $M=0.5$ flow. This numerical example is devoted to check the ability of the method to simulate long distance wave propagation. We follow the setup of this case presented in [6,41]. The source term is included by defining the vector $\boldsymbol{S}$ in Eq. (1) as

$$
\boldsymbol{S}(\boldsymbol{r}, t)=\frac{\epsilon}{h} e^{-\kappa r^{2}} \sin (\omega t)(1,0,0,1)^{T}
$$

with $\epsilon=0.5, \kappa=\ln (2) / 2, \omega=\frac{2 \pi}{20}$ and $r$ indicates the distance to the initial location of the pulse. The angular frequency is $2 \pi / 30$ and the wavelength is $\lambda=30$. The computational domain of interest is a circle with radius $R=100$. In order to damp the waves leaving the domain we have added an absorbing layer, which is placed from the boundary of the computational domain (the circular area defined by $R=100$ )to $x= \pm 300$ and $y= \pm 300$. We solve this test case using a random distribution of 45.111 particles. The configuration of the computational domain and the absorbing layer is shown in Fig. 19. 

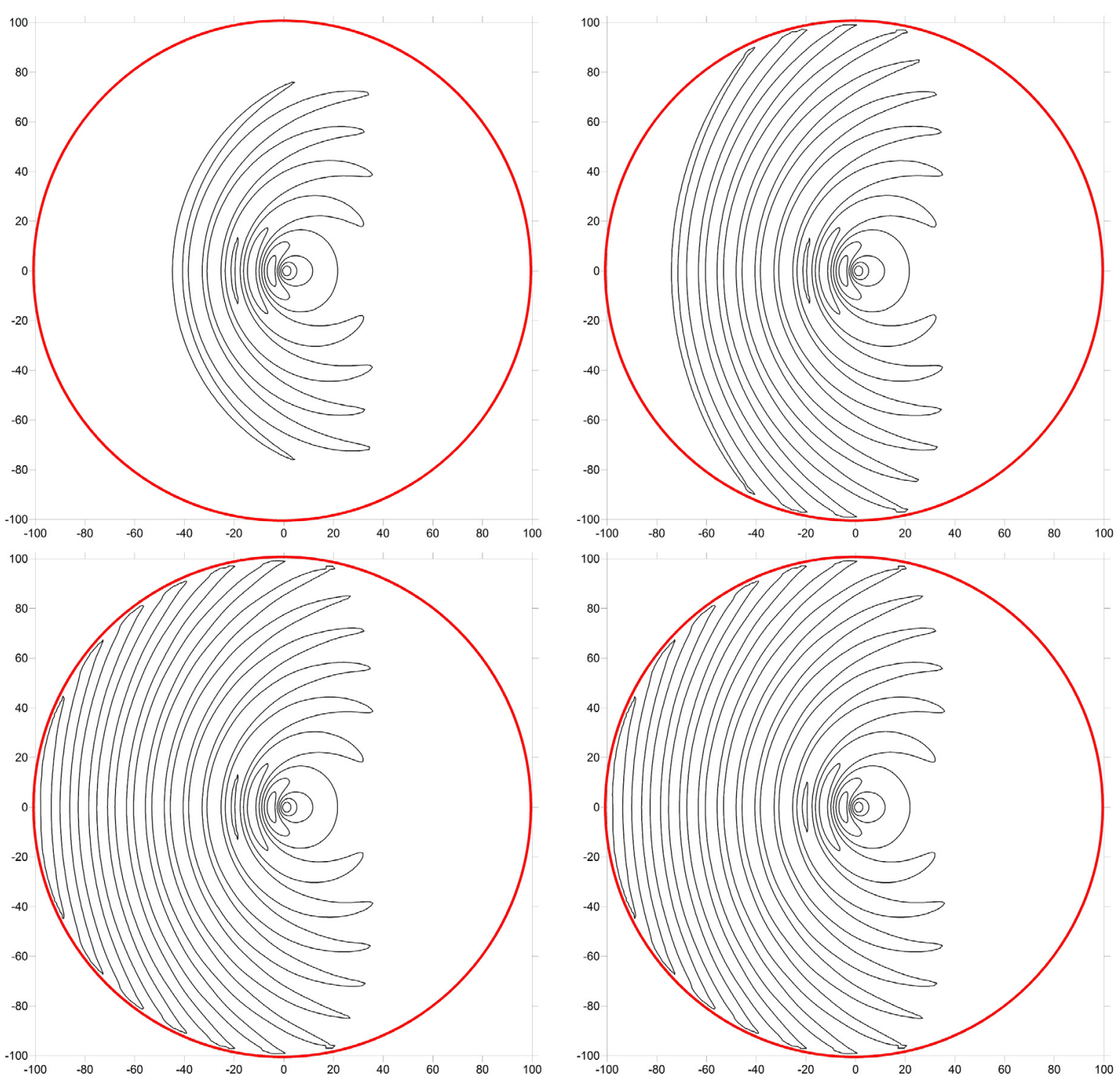

Fig. 20. Monopole source radiation in uniform $M=0.5$ mean flow. Acoustic pressure field at $t=90, t=150, t=210, t=270$.

The acoustic pressure field for $t=90, t=150, t=210$ and $t=270$ is shown in Fig. 20. Due the imposed mean flow, the apparent wavelength is different upstream and downstream the source. The acoustic waves propagate upstream and downstream at the velocity $1 \pm M$ with apparent wavelengths of $\lambda_{c}=(1 \pm M) \lambda$. As shown in these figures and also in Fig. 21, no spurious oscillations appear and the numerical solution matches perfectly with the analytic solution as it is presented in Fig. 22 for $t=270$. The analytical solution is obtained by a convolution product of the source term with the 2-D Green function as indicated in [6].

\subsection{Acoustic wave scattering by a circular cylinder}

The last benchmark case is the acoustic wave scattering by a circular cylinder, which is a widely used validation case $[39,44,45]$. The problem setup is schematically presented in Fig. 23, where a circular cylinder with radius $R=0.5$ is located at the center of the computational domain. A source of noise is placed at $(x, y)=(4,0)$. This setup is a 


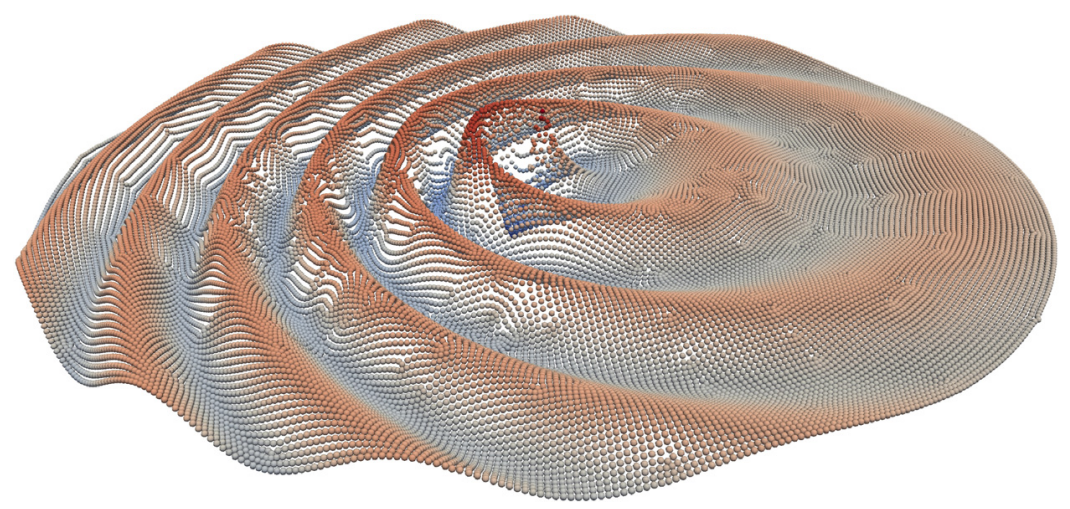

Fig. 21. Monopole source radiation in uniform $M=0.5$ mean flow Acoustic pressure field at $t=270$ in the computational domain (absorbing layer region is not shown). It is observed the absence of spurious noise.

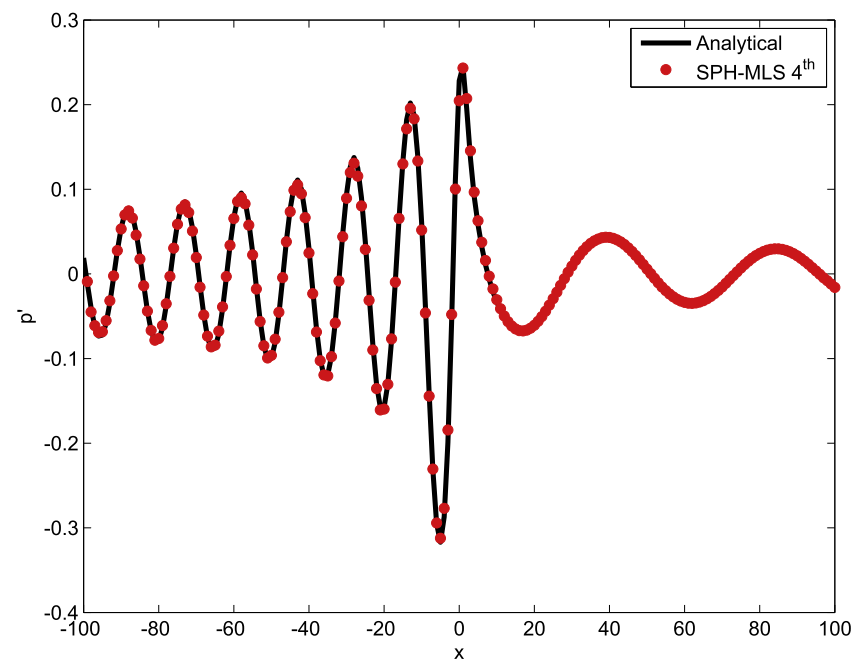

Fig. 22. Monopole source radiation in uniform $M=0.5$ mean flow Acoustic pressure field along $y=0$ at $t=270$. Comparison with the analytical solution.

simplified model of the propagation of the noise generated at an aircraft turbine engine impinging on the fuselage [44]. Points labeled as $A, B, C, D$ are measurement points for the pressure field.

In order to avoid spurious reflections at boundaries an absorbing layer is also used here, which is placed from $R=6.5$ to $R=15$.

In this numerical example we consider two cases, depending on the source of noise selected. First, we chose to place a Gaussian pulse and then, a more difficult problem is performed by using a time-periodic Gaussian source.

\subsubsection{Gaussian pulse scattering by a circular cylinder}

First, we choose to use a very simple source of noise, in order to perform a first test of the ability of the numerical scheme to model the interaction of the noise with a curved surface. In this case, the initial condition for the pulse reads

$$
\rho^{\prime}(x, y, t=0)=e^{-\kappa r^{2}}, \quad u^{\prime}=v^{\prime}=0, \quad p^{\prime}(x, y, t=0)=e^{-\kappa r^{2}}
$$

with $\kappa=\ln (2) / b^{2}, b=0.2$ and $r$ indicates the distance to the initial location of the pulse, which is located at $(x, y)=(4,0)$. In this numerical example, the particles are equidistantly distributed with $\Delta X=0.04$ on the computational domain. The distribution of particles for the computational domain of interest and the absorbing layer 


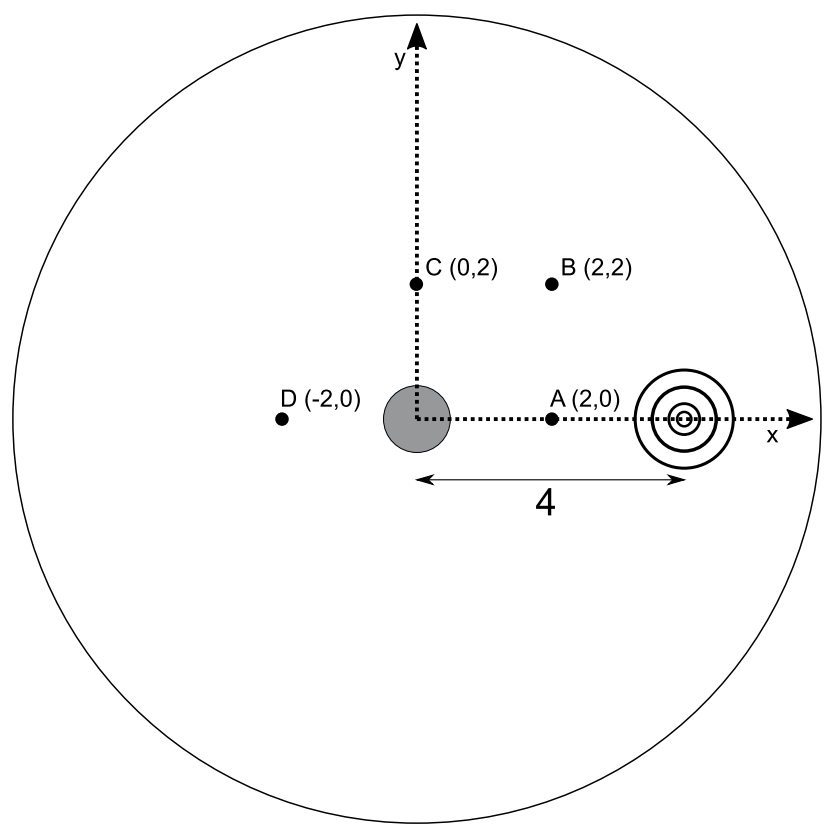

Fig. 23. Acoustic wave scattering by a circular cylinder. Schematic setup of the problem.

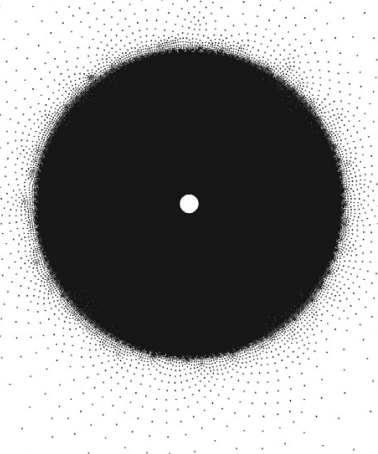

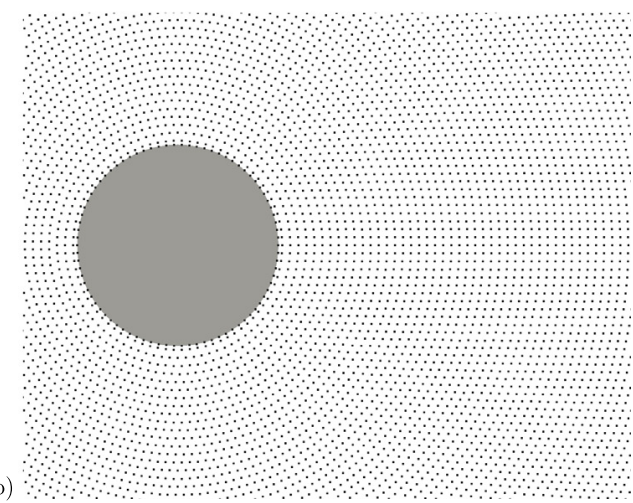

b)

Fig. 24. Acoustic wave scattering by a circular cylinder: Gaussian Pulse. (a) Particle distribution including the absorbing layer and (b) close view of the particle distribution around the cylinder.

is presented in Fig. 24. This configuration leads to a distribution of 111.403 particles. Note that this resolution is similar to that used in $[44,45]$ for optimized high-order finite difference schemes.

In Fig. 25, pressure contours are shown at different times. We note that no spurious noise appears and the results are in agreement with those of $[44,45]$.

The time history of pressure fluctuations at measurement points $A, B, C, D$, located as indicated in Fig. 23, are shown in Fig. 26 compared with the analytical solution [39]. Again, no discrepancies are observed, and the numerical solution perfectly matches the analytical solution. 


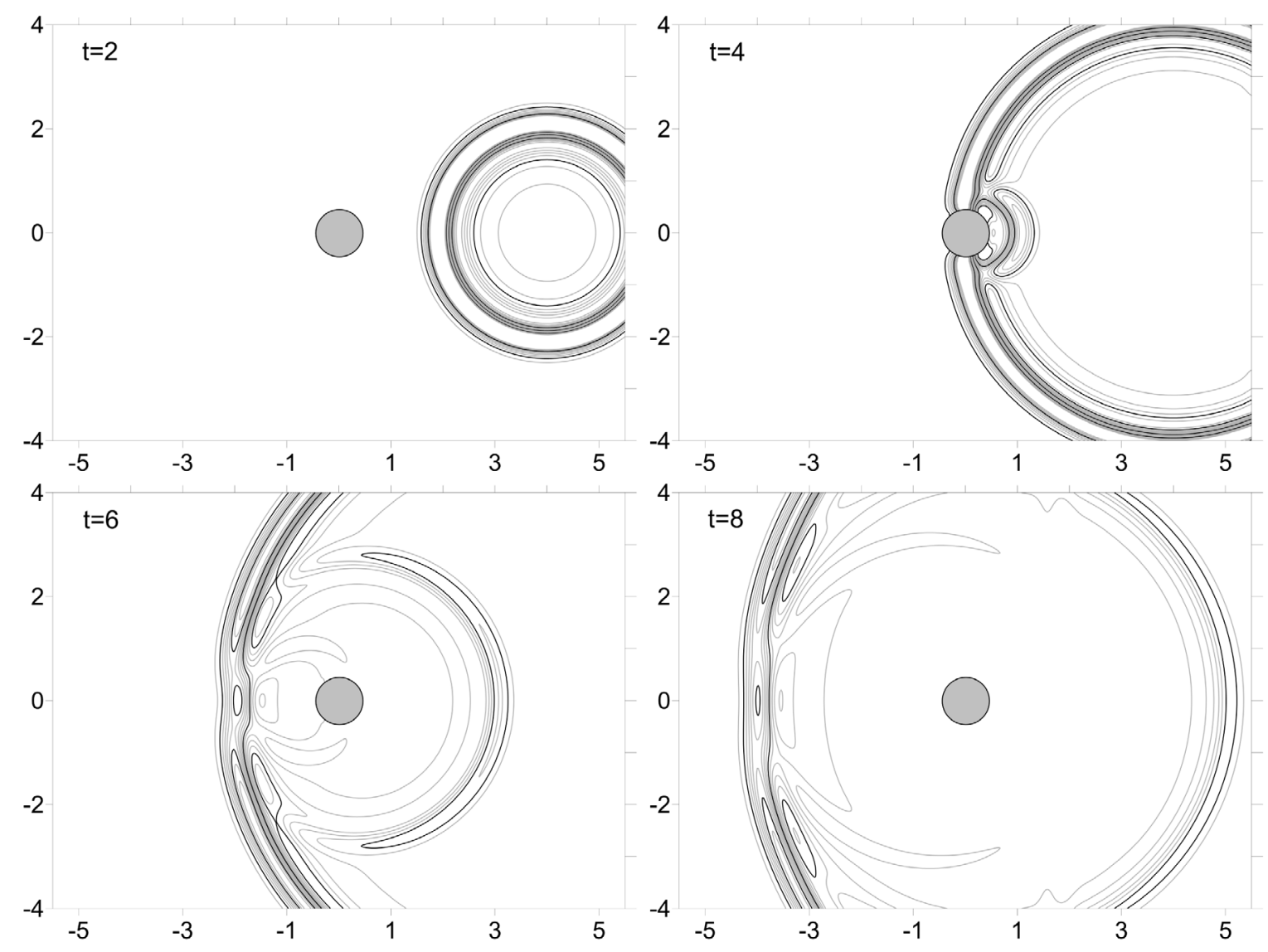

Fig. 25. Acoustic wave scattering by a circular cylinder: Gaussian Pulse. Pressure fluctuations contours at times $t=2,4,6,8$.

\subsubsection{Time-periodic Gaussian source scattering by a circular cylinder}

In the second benchmark of acoustic scattering by a circular cylinder, we use a periodic source of noise instead of a simple pulse, defined as

$$
\boldsymbol{S}(\boldsymbol{r}, t)=e^{-\kappa r^{2}} \sin (\omega t)(1,0,0,1)^{T}
$$

with $\kappa=\ln (2) / b^{2}, b=0.2, \omega=8 \pi$ and $r$ indicates the distance to the initial location of the pulse, which is also located at $(x, y)=(4,0)$. Two different particle distributions are used in this example. The first distribution, labeled as $M 1$, has a total number of 356.848 particles and the second, $M 2$, has a total number of 1.344 .563 particles, which correspond approximately to 12.5 and 25 points per wave, respectively. Similar spatial resolution has been used for other authors using other numerical schemes [44,45].

This test case is one of the most difficult benchmark problems for validation of computational codes for CAA which aims to test the long-time stability of the numerical scheme and also its suitability of far-field treatments in presence of scattering bodies [46].

The obtained acoustic field around the cylinder is plotted in Fig. 27.

In Fig. 28 the Root Mean Square (RMS) of the pressure fluctuation is shown at $r=0.52$ for both particles resolution and compared with the analytical solution, which is computed as described in [39].

The results obtained for the proposed method match very well with the analytical solution, and they are comparable to those obtained in the literature [45] for a fourth-order dispersion-relation-preserving finite difference scheme. 

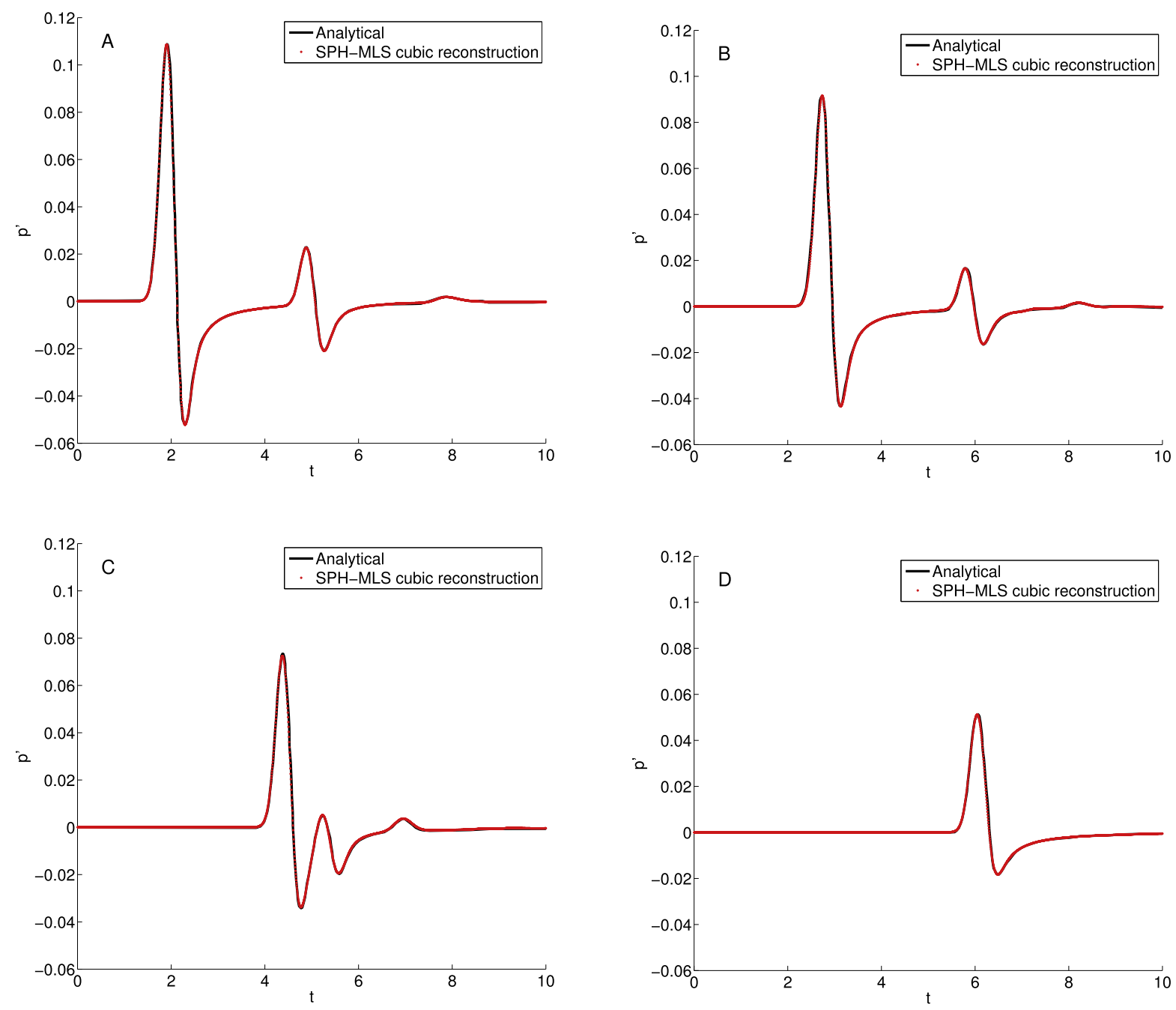

Fig. 26. Acoustic wave scattering by a circular cylinder: Gaussian Pulse. Time histories of pressure fluctuations at locations $A(2,0), B(2,2)$, $C(0,2)$ and $D(-2,0)$, as indicated at Fig. 23.

\section{Conclusions}

In this work, we propose a new meshless approach based on a Galerkin discretization of a set of conservation equations on an Arbitrary Lagrangian-Eulerian framework, using Riemann solvers for the discretization of the convective fluxes. The proposed method is applied to the Linearized Euler Equations to solve Computational Aeroacoustic problems. The formulation uses MLS as weight functions, and can be applied in both, Lagrangian and Eulerian configurations. The use of MLS allows for a reduced stencil in the computations, verifies the discrete partition of unity and also reproduces the zero-gradient condition for constant functions.

Some CAA benchmark problems has been addressed to show the accuracy of the proposed formulation, even in presence of solid walls. The performed numerical tests show that the proposed methodology can be applied to CAA problems, where high accurate numerical methods are demanded.

\section{Acknowledgments}

This work has been partially supported by the Ministerio de Economía y Competitividad (grant \#DPI2015-68431$\mathrm{R}$ ) of the Spanish Government and by the Consellería de Educación e Ordenación Universitaria of the Xunta de Galicia (grant \#GRC2014/039), cofinanced with FEDER funds and the Universidade da Coruña. 


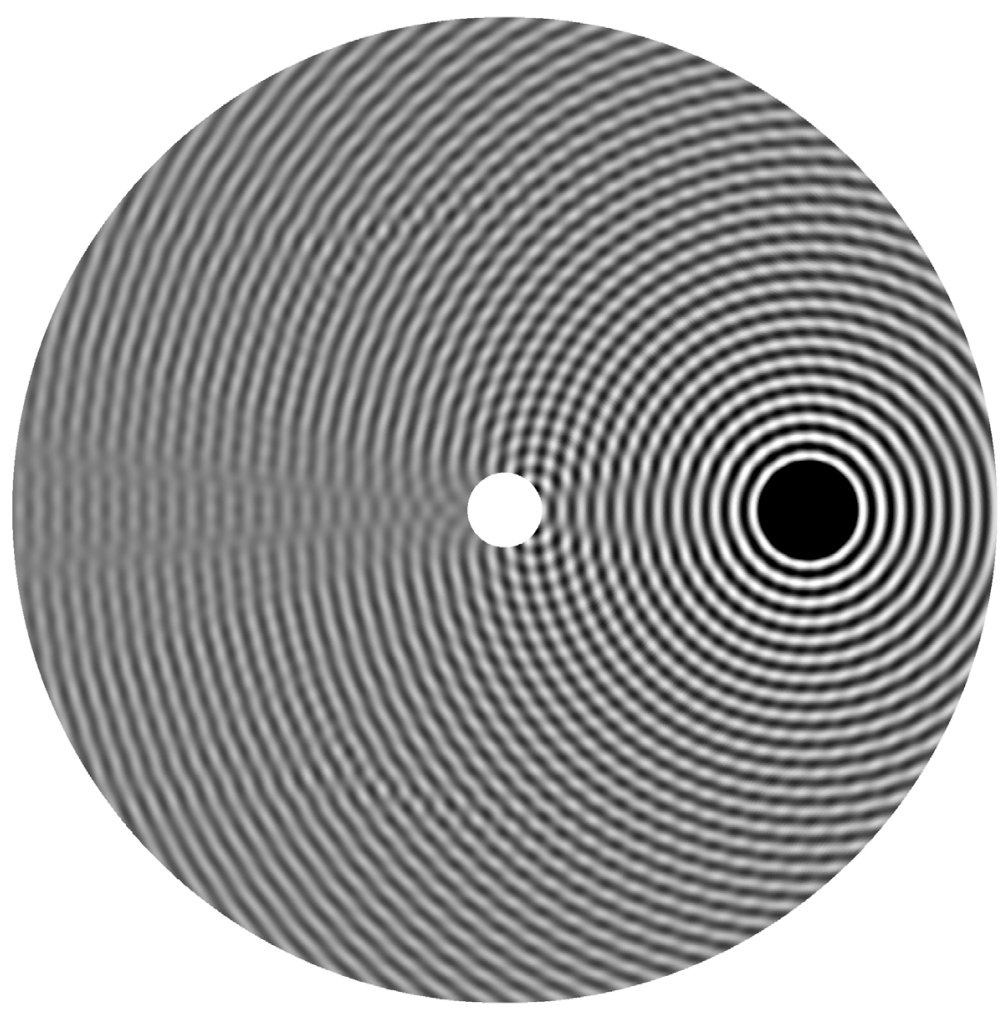

Fig. 27. Acoustic wave scattering by a circular cylinder: Time-periodic Gaussian Source. Pressure fluctuations contours for $M 2$ particle distribution.

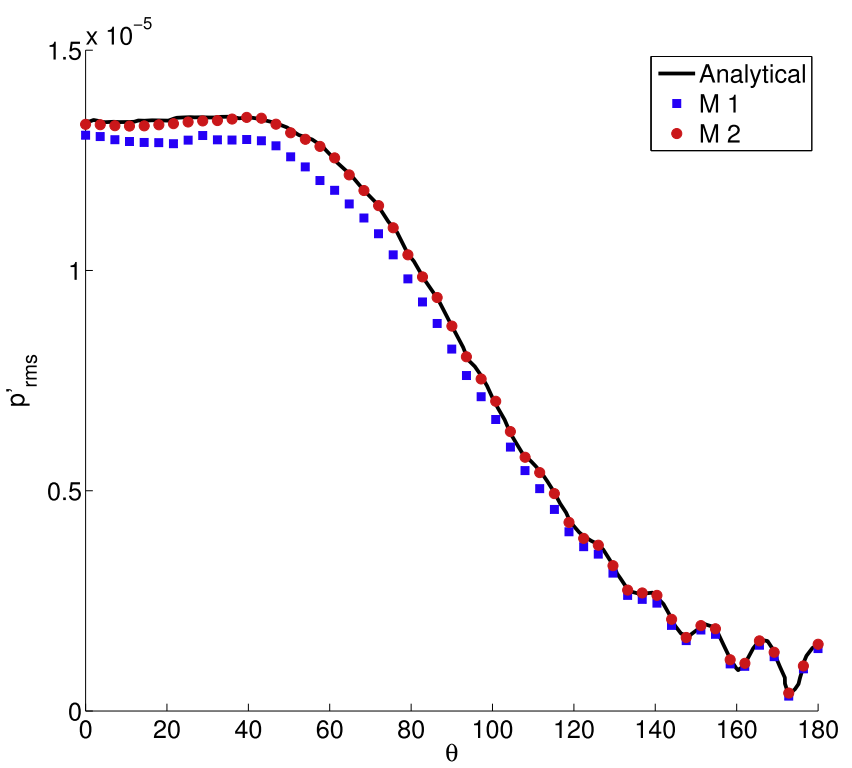

Fig. 28. Acoustic wave scattering by a circular cylinder: Time-periodic Gaussian Source. Comparison of the RMS of pressure fluctuations at $r=0.52$ for two different particle distribution $(M 1=356.848$ and $M 2=1.344 .563$ particles $)$ and the analytical solution. 


\section{References}

[1] C.K.W. Tam, Computational acoustics: Issues and methods, AIAA J. 33 (10) (1995) 1788-1796.

[2] F.Q. Hu, M.Y. Hussaini, J.L. Manthey, A low-dissipation and low dispersion Runge-Kutta Scheme for Computational Acoustics, J. Comput. Phys. 124 (1996) 177-191.

[3] C.K.W. Tam, J.C. Webb, Dispersion-relation-preserving finite difference schemes for computational acoustics, J. Comput. Phys. 107 (1993) $262-281$

[4] S.K. Lele, Compact finite difference schemes with spectral-like resolution, J. Comput. Phys. 103 (1992) 16-42.

[5] C. Bogey, C. Bailly, A family of low dispersive and low dissipative explicit schemes for flow and noise computations, J. Comput. Phys. 194 (1) (2004) 194-214.

[6] C. Bailly, D. Juvé, Numerical solution of acoustic propagation problems using linearized Euler equations, AIAA J. 38 (1) (2000) $22-29$.

[7] F. Daude, J. Berland, T. Emmert, P. Lafon, F. Crouzet, C. Bailly, A high-order finite-difference algorithm for direct computation of aerodynamic sound, Comput. \& Fluids 61 (2012) 46-63.

[8] K. Li, Q.B. Huang, J.L. Wang, L.G. Lin, An improved localized radial basis function meshless method for computational aeroacoustics, Eng. Anal. Bound. Elem. 35 (2011) 47-55.

[9] S. Wang, S. Li, Q. Huang, K. Li, An improved collocation meshless method based on the variable shaped radial basis function for the solution of the interior acoustic problems, Math. Probl. Eng. 2012 (2012). http://dx.doi.org/10.1155/2012/632072. Article ID 632072, 20 pages.

[10] P.R.S. Antunes, S.S. Valtchev, A meshfree numerical method for acoustic wave propagation problems in planar domains with corners and cracks, J. Comput. Appl. Math. 234 (9) (2010) 2646-2662.

[11] J. Bajko, L. Čermák, M. Jícha, High order finite point method for the solution to the sound propagation problems, Comput. Methods Appl Mech. Engrg. 280 (2014) 157-175.

[12] Y.O. Zhang, T. Zhang, H. Ouyang, T.Y. Li, SPH simulation of acoustic waves: Effects of frequency, sound pressure, and particle spacing, Math. Probl. Eng. 2015 http://dx.doi.org/10.1155/2015/348314. Article ID 348314, 7 pages.

[13] Y.O. Zhang, T. Zhang, H. Ouyang, T.Y. Li, Efficient SPH simulation of time-domain acoustic wave propagation, Eng. Anal. Bound. Elem. 62 (2016) 112-122.

[14] C.T. Wolfe, S.K. Semwal, Acoustic modeling of reverberation using smoothed particle hydrodynamics, in: 16th International Conference in Central Europe on Computer Graphics, Visualization and Computer Vision, WSCG'2008 - In Co-operation with EUROGRAPHICS, Full Papers, 2008, pp. 191-198.

[15] P. Hahn, D. Negrut, On the use of meshless methods in acoustic simulations, ASME Int. Mech. Eng. Congr. Expo., Proc. 13 (2010) 185-199.

[16] P. Lancaster, K. Salkauskas, Surfaces generated by moving least squares methods, Math. Comp. 37 (155) (1981) 141-178.

[17] W.K. Liu, W. Hao, Y. Chen, S. Jun, J. Gosz, Multiresolution reproducing Kernel Particle Methods, Comput. Mech. 20 (1997) 295-309.

[18] J.P. Vila, On particle weighted methods and smooth particle hydrodynamics, Math. Models Methods Appl. Sci. 9 (2) (1999) $161-209$.

[19] D. Avesani, M. Dumbser, A. Bellin, A new class of moving-least-squares WENO-SPH schemes, J. Comput. Phys. 270 (2014) $278-299$.

[20] X. Nogueira, L. Ramírez, Stéphane. Clain, R. Loubère, L. Cueto-Felgueroso, I. Colominas, High accurate SPH method with multidimensional optimal order detection, Comput. Methods Appl. Mech. Engrg. 310 (2016) 134-155.

[21] E. Oñate, S. Idelsohn, O.C. Zienkiewicz, R.L. Taylor, A finite point method in computational mechanics applications to convective transport and fluid flow, Internat. J. Numer. Methods Engrg. 39 (1996) 3839-3866.

[22] E. Oñate, Derivation of stabilized equations for numerical solution of advective-diffusive transport and fluid flow problems, Comput. Methods Appl. Mech. Engrg. 151 (1998) 233-265.

[23] E. Ortega, E. Oñate, S. Idelsohn, A finite point method for adaptive three-dimensional compressible flow calculations, Internat. J. Numer. Methods Fluids 60 (2009) 937-971.

[24] E. Gaburov, K. Nitadori, Astrophysical weighted particle magnetohydrodynamics, Mon. Not. R. Astron. Soc. 414 (2011) $129-154$.

[25] P.F. Hopkins, A new class of accurate, mesh-free hydrodynamic simulation methods, Mon. Not. R. Astron. Soc. 450 (1) (2015) 53-110.

[26] B. Ben Moussa, On the convergence of SPH method for scalar conservation laws with boundary conditions, Methods Appl. Anal. 13 (2006) $29-62$.

[27] R.A. Gingold, J.J. Monaghan, Smoothed particle hydrodynamics-theory and application to non-spherical stars, Mon. Not. R. Astron. Soc. 181 (1977) 375-389.

[28] J.G. Wang, G.R. Liu, A point interpolation meshless method based on radial basis functions, Internat. J. Numer. Methods Engrg. 54 (2002) $1623-1648$.

[29] M. Arroyo, M. Ortiz, Local maximum-entropy approximation schemes: A seamless bridge between finite elements and meshfree methods, Internat. J. Numer. Methods Engrg. 65 (2005) 2167-2202.

[30] L. Traversoni, Natural neighbor finite elements, in: International Conference on Hydraulic Engineering Software Hydrosoft Proceedings, vol. 2, Computational Mechanics Publications, 1994, pp. 291-297 65.

[31] J. Braun, M. Sambridge, A numerical method for solving partial differential equations on highly irregular evolving grids, Nature 376 (1995) $655-660$.

[32] L. Cueto-Felgueroso, I. Colominas, X. Nogueira, F. Navarrina, M. Casteleiro, Finite volume solvers and moving least-squares approximations for the compressible Navier-Stokes equations on unstructured grids, Comput. Methods Appl. Mech. Engrg. 196 (2007) $4712-4736$.

[33] S. Khelladi, X. Nogueira, F. Bakir, I. Colominas, Toward a higher-order unsteady finite volume solver based on reproducing Kernel particle method, Comput. Methods Appl. Mech. Engrg. 200 (2011) 2348-2362.

[34] J.J. Monaghan, Smoothed particle hydrodynamics, Annu. Rev. Astron. Astrophys. 30 (1992) 543-574.

[35] H. Wendland, Piecewise polynomial, positive definite and compactly supported radial functions of minimal degree, Adv. Comput. Math. 4 (1995) 389-396. 
[36] S. Rosswog, Boosting the accuracy of SPH techniques: Newtonian and special-relativistic tests, Mon. Not. R. Astron. Soc. 448 (2015) $3628-3664$.

[37] V.V. Rusanov, The calculation of the interaction of non-stationary shock waves and obstacles, USSR Comput. Math. Math. Phys. 1 (2) (1962) 304-320.

[38] G.A. Dilts, Moving-least-squares-particle hydrodynamics. Consistency and Stability, Internat. J. Numer. Methods Engrg. 44 (1999) $1115-1155$.

[39] C.K.W. Tam, J.C. Hardin, Second computational aeroacoustics (CAA) workshop on benchmark problems, (1997) NASA-CP-3352.

[40] J.C. Hardin, J.R. Ristorcelli, C.K.W. Tam, ICASE/LaRC Workshop on Benchmark Problems in Computational Aeroacoustics, NASA Conference Publication, 19953300.

[41] F.Q. Hu, On constructing stable perfectly matched layers as an absorbing boundary condition for Euler equations, AIAA Pap. (2003) 2003-3301.

[42] X. Nogueira, I. Colominas, L. Cueto-Felgueroso, S. Khelladi, F. Navarrina, M. Casteleiro, Resolution of computational aeroacoustics problems on unstructured grids with a higher-order finite volume scheme, J. Comput. Appl. Math. 234 (7) (2010) 2089-2097.

[43] M. Popescu, Cartesian cut-cell method with local grid refinement for wave computations, AIAA Pap. 200 (2006) $2006-2522$.

[44] J.H. Seo, R. Mittal, A high-order immersed boundary method for acoustic wave scattering and low-Mach number flow-induced sound in complex geometries, J. Comput. Phys. 230 (2011) 1000-1019.

[45] X. Sun, Y. Jiand, A. Liang, X. Jing, An immersed boundary computational model for acoustic scattering problems with complex geometries, J. Acoust. Soc. Am. 132 (2012) 3190-3199.

[46] NASA, Fourth computational aeroacoustics (CAA) workshop on benchmark problems, in: Tech. Rep., 2004, NASA/CP-2004-212954. 